\title{
Statistical Comparison of Regional Atmospheric Modeling System Forecast Meteorology with Observations (U)
}

\author{
Robert L. Buckley, Allen H. Weber and Jilene H. Weber
}

Savannah River Technology Center

Publication Date: December 2001

Westinghouse Savannah River Company

Savannah River Site

Aiken, SC 29808

This document was prepared in connection with work done under Contract No. DE-AC09-96SR18500

with the U.S. Department of Energy 
This document was prepared in conjunction with work accomplished under Contract No. DE-AC09-96SR18500 with the U.S. Department of Energy.

\section{DISCLAIMER}

This report was prepared as an account of work sponsored by an agency of the United States Government. Neither the United States Government nor any agency thereof, nor any of their employees, makes any warranty, express or implied, or assumes any legal liability or responsibility for the accuracy, completeness, or usefulness of any information, apparatus, product or process disclosed, or represents that its use would not infringe privately owned rights. Reference herein to any specific commercial product, process or service by trade name, trademark, manufacturer, or otherwise does not necessarily constitute or imply its endorsement, recommendation, or favoring by the United States Government or any agency thereof. The views and opinions of authors expressed herein do not necessarily state or reflect those of the United States Government or any agency thereof.

This report has been reproduced directly from the best available copy.

Available for sale to the public, in paper, from: U.S. Department of Commerce, National Technical Information Service, 5285 Port Royal Road, Springfield, VA 22161

phone: (800) 553-6847

fax: (703) 605-6900

email: orders@ntis.fedworld.gov

online ordering: http://www.ntis.gov/support/index.html

Available electronically at http://www.doe.gov/bridge

Available for a processing fee to U.S. Department of Energy and its contractors, in paper, from: U.S. Department of Energy, Office of Scientific and Technical Information, P.O. Box 62, Oak Ridge, TN 37831-0062

phone: (865)576-8401

fax: $(\mathbf{8 6 5}) 576-5728$

email: reportseadonis.osti.gov 
WSRC-TR-2001-00563

December 2001

DOCUMENT: $\quad$ WSRC-TR-2001-00563

TITLE:

Statistical Comparison of Regional Atmospheric Modeling System Forecast Meteorology with Observations (U)

TASK:

TECHNICAL REVIEW

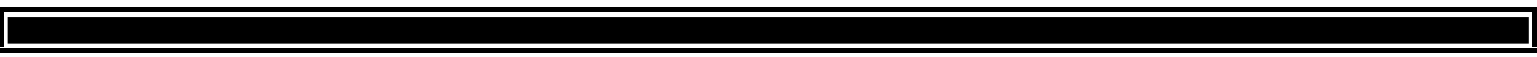

B. L. O'Steen

Date:

SRTC/Measurement Technology Department/Nonproliferation Technologies Section

\section{APPROVALS}

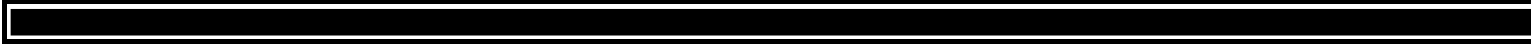

R. P. Addis, Manager, ATG

Date:

SRTC/Measurement Technology Department/Nonproliferation Technologies Section

A. L. Boni, Manager, NTS

Date:

SRTC/Measurement Technology Department/Nonproliferation Technologies Section 
WSRC-TR-2001-00563

December 2001

(Blank Page) 


\begin{abstract}
A statistical comparison of observations and forecasts using the Regional Atmospheric Modeling System (RAMS) for operations at the Savannah River Site (SRS) is discussed. Simulated and observed values of wind direction, wind speed, and temperature, collected twice daily for a twoyear period from April 1998 through March 2000, are compared in a variety of ways for 5 different locations in the southeast United States. Turbulence quantities are also compared for a one-year period beginning in February 1999 for the SRS. Results are presented in the form of scatter plots and histograms, as well as time-based line plots for the different locations within the modeling domain. Both surface and upper-level model predictions are compared with observations taken from both the National Weather Service and local SRS tower locations (surface measures only). Variability based on the time of year, the forecast hour, the location of the observations, and the height above ground for each of these variables is discussed. Statistics of accuracy used for comparison include absolute mean bias, relative bias, root-mean-square error, standard deviation, and the index of agreement. The most severe degradation in results occurs during the transition periods of early evening ( $\sim 19$ to 20 LST) and late morning ( $\sim 07$ to 08 LST), especially for temperature.
\end{abstract}


WSRC-TR-2001-00563

December 2001

\section{TABLE OF CONTENTS}

1. INTRODUCTION

2. BACKGROUND 3

2.1 Prognostic Model (RAMS) 3

$\begin{array}{ll}\text { 2.2 Modifications to RAMS } & 4\end{array}$

$\begin{array}{ll}2.3 \text { Observations } & 5\end{array}$

$\begin{array}{ll}2.4 \text { Statistical Comparisons } & 6\end{array}$

3. RESULTS 10

$\begin{array}{ll}3.1 \text { Ensemble Averages } & 10\end{array}$

$\begin{array}{ll}\text { 3.2 Time Series of Statistics } & 11\end{array}$

3.3 Upper-air 12

3.4 Skill 12

4. DISCUSSIONS

$\begin{array}{ll}\text { 4.1 Ensemble Averages } & 13\end{array}$

$\begin{array}{ll}\text { 4.2 Time Series of Statistics } & 15\end{array}$

$\begin{array}{ll}4.3 \text { Upper-air } & 15\end{array}$

$\begin{array}{ll}\text { 4.4 Skill } & 17\end{array}$

$\begin{array}{lr}\text { 5. CONCLUSIONS } & 18\end{array}$

$\begin{array}{ll}\text { REFERENCES } & 22\end{array}$ 


\section{LIST OF TABLES}

Table 1: Sensitivity of Wind Speed Differences to Surface Roughness, $z_{0}$. 26

Table 2: Wind Direction Comparisons at the Surface. 27

Table 3: Wind Speed Comparisons at the Surface. 28

Table 4: Temperature Comparisons at the Surface. 29

Table 5: Wind Direction Comparisons at Upper-Air Levels. 30

Table 6: Wind Speed Comparisons at Upper-Air Levels. 31

Table 7: Temperature Comparisons at Upper-Air Levels. 32

\section{LIST OF FIGURES}

Figure 1: RAMS domain used in the applications at the SRS. The five cities from which statistical comparisons are made (along with the SRS) are indicated. Upper-air comparisons are also made at Atlanta and Charleston.

Figure 2: Histogram plots showing wind direction (deg) comparisons for all six locations at the surface for a forecast period of: (a) 0-hr, (b) 6-hr, (c) 12-hr, (d) 18-hr, (e) 24-hr. For each forecast period, an absolute difference constrained to be between 0 and $180^{\circ}$ is calculated and the percentage of bin values in degrees is shown. The cumulative amount for a given error range is given by the dash-dot line connecting the various bin levels. In addition, "No.Pts." is the number of times represented in the plot, while "AbsBias" indicates the mean absolute difference for that particular forecast time.

Figure 3: Scatter plot showing surface wind speed $\left(\mathrm{m} \mathrm{s}^{-1}\right)$ comparisons for the six locations for a forecast period of: (a) 0-hr, (b) 6-hr, (c) 12-hr, (d) 18-hr, (e) 24-hr. The observed quantity is shown along the $x$-axis, and the simulated quantity is shown along the $y$-axis. The bracketing value is $1 \mathrm{~m} \mathrm{~s}^{-1}$ to either side of the $45^{\circ}$ line. For each forecast period, the following are given: "No.Pts.", the number of times represented in the plot, "Mean", the mean difference between simulated and observed wind speed, "St.Dev", the standard deviation, "AbsBias", the mean absolute bias, and the percentage of times in which the simulated wind speed was within $\pm 1 \mathrm{~m} \mathrm{~s}^{-1}$ of the observed value.

Figure 4: Scatter plot showing surface deviation in azimuth angle (deg) comparisons for the SRS for a forecast period of: (a) 0-hr, (b) 6-hr, (c) 12-hr, (d) 18-hr, (e) 24-hr. The observed quantity is shown along the $x$-axis, and the simulated quantity is shown along the $y$-axis. The bracketing value is $5^{\circ}$ to either side of the $45^{\circ}$ line. For each forecast 
period, the following are given: "No.Pts.", the number of times represented in the plot, "Mean", the mean difference between simulated and observed $\sigma_{A}$, "St.Dev.", the standard deviation, "AbsBias", the mean absolute bias, and the percentage of times in which the simulated $\sigma_{A}$ was within $\pm 5^{\circ}$ of the observed value.

Figure 5: Scatter plot showing surface deviation in elevation angle (deg) comparisons for the SRS for a forecast period of: (a) 0-hr, (b) 6-hr, (c) 12-hr, (d) 18-hr, (e) 24-hr. The observed quantity is shown along the $x$-axis, and the simulated quantity is shown along the $y$-axis. The bracketing value is $2.5^{\circ}$ to either side of the $45^{\circ}$ line. For each forecast period, the following are given: "No.Pts.", the number of times represented in the plot, "Mean", the mean difference between simulated and observed $\sigma_{E}$, "St.Dev.", the standard deviation, "AbsBias", the mean absolute bias, and the percentage of times in which the simulated $\sigma_{E}$ was within $\pm 2.5^{\circ}$ of the observed value.

Figure 6: Scatter plot showing surface temperature $(\mathrm{K})$ comparisons for the six locations for a forecast period of: (a) 0-hr, (b) 6-hr, (c) 12-hr, (d) 18-hr, (e) 24-hr. The observed quantity is shown along the $x$-axis, and the simulated quantity is shown along the $y$-axis. The bracketing value is $2 \mathrm{~K}$ to either side of the $45^{\circ}$ line. For each forecast period, the following are shown: "No.Pts.", the number of times represented in the plot, "Mean", the mean difference between simulated and observed temperature, "St.Dev.", the standard deviation, "AbsBias", the mean absolute bias, and the percentage of times in which the simulated temperature was within $\pm 2 \mathrm{~K}$ of the observed value.

Figure 7: Plots of absolute bias (wind direction) or mean difference (wind speed, temperature) as a function of the time of day in the forecast for Augusta, Georgia. In this case, simulations using the analysis valid for a time of 00 GMT are used to generate a forecast. Note that $12 \mathrm{GMT}$ is an early morning period for this location. Each line represents an average over all simulations within a given month (24 months in all) for the resulting difference between simulation and observation. Solid lines are from the year 1998, dotted lines from 1999, and dashed lines from 2000. Note that for the mean differences, a positive number denotes the simulation over-predicted the result.

Figure 8: Comparison of simulated and observed wind direction for all 5 cities at the surface for forecast periods of 0,12 , and 24 hours, as well as for the SRS climatology location (from February 1999 onward). Each line in each plot represents a different location, as denoted in the legend. Each symbol represents the average for a particular month, beginning at the far left with April 1998. In this case, the absolute difference in simulation and observation (constrained to $\leq 180^{\circ}$ ) over that month for that particular forecast time is shown. A mean absolute difference is also shown as averaged over all locations and times, with a numerical value given in parentheses near the center of each row. 
Figure 9: Comparison of simulated and observed wind speed for all 5 cities at the surface for forecast periods of 0,12 , and 24 hours, as well as for the SRS climatology location (from February 1999 onward). Each line in each plot represents a different location, as denoted in the legend. Each symbol represents the average for a particular month, beginning at the far left with April 1998. The left-hand column shows the absolute difference in simulation and observation over that month for that particular forecast time. The righthand column shows mean relative differences between simulation and observation. Positive numbers denote the simulation over-predicted the result. A mean absolute difference is also shown for the left-hand column as averaged over all locations and times, with a numerical value given in parentheses near the center of each row.

Figure 10: Comparison of simulated and observed deviations in azimuth and elevation angle at the surface at SRS for forecast periods of 0, 12, and 24 hours. Format as in Fig. 9, except the solid line is $\sigma_{A}$ and the dotted line is $\sigma_{E}$. The mean absolute differences are in italics for $\sigma_{A}$, and in normal text for $\sigma_{E}$.

Figure 11: Comparison of simulated and observed temperature for all 5 cities at the surface for forecast periods of 0,12 , and 24 hours, as well as for the SRS climatology location (from February 1999 onward). Format as in Fig. 9.

Figure 12: As in Figure 8, except comparisons of simulated and observed wind direction are for Atlanta and Charleston at the 700-mb pressure level, using observations taken from upper-air reports.

Figure 13: As in Figure 9, except comparisons of simulated and observed wind speed are for Atlanta and Charleston at the 500-mb pressure level, using observations taken from upper-air reports.

Figure 14: As in Figure 11, except comparisons of simulated and observed temperature are for Atlanta and Charleston at the $300-\mathrm{mb}$ pressure level, using observations taken from upper-air reports.

Figure 15: Plots of the ratio of root-mean-square error to observed standard deviation as a function of the forecast time for the six different locations. Each value at a given forecast time is averaged over all months from April 1998 to March 2000. The top panels are for vector winds, while the bottom panels are for temperature.

Figure 16: Plots of the ratio of root-mean-square error to observed standard deviation as a function of the month for the six different locations. Each value for a given month is averaged over all forecast times from 0 to 24 hours at 2-hr increments. The top panels are for vector winds, while the bottom panels are for temperature.

Figure 17: Plots of the ratio of unbiased to biased root-mean-square error as a function of the forecast time for the six different locations. Each value at a given forecast time is 
averaged over all months from April 1998 to March 2000. The top panels are for vector winds, while the bottom panels are for temperature.

Figure 18: Plots of the ratio of unbiased to biased root-mean-square error as a function of the month for the six different locations. Each value for a given month is averaged over all forecast times from 0 to 24 hours at 2 -hr increments. The top panels are for vector winds, while the bottom panels are for temperature.

Figure 19: Plots of the ratio of simulated to observed standard deviation as a function of the forecast time for the six different locations. Each value at a given forecast time is averaged over all months from April 1998 to March 2000. The top panels are for vector winds, while the bottom panels are for temperature.

Figure 20: Plots of the ratio of simulated to observed standard deviation as a function of the month for the six different locations. Each value for a given month is averaged over all forecast times from 0 to 24 hours at 2 -hr increments. The top panels are for vector winds, while the bottom panels are for temperature.

Figure 21: Plots of the index of agreement as a function of the forecast time for the six different locations. Each value at a given forecast time is averaged over all months from April 1998 to March 2000 The top panels are for vector winds, while the bottom panels are for temperature.

Figure 22: Plots of the index of agreement as a function of the month for the six different locations. Each value for a given month is averaged over all forecast times from 0 to 24 hours at 2-hr increments. The top panels are for vector winds, while the bottom panels are for temperature. 


\section{INTRODUCTION}

The Savannah River Site (SRS) is a Department of Energy (DOE) complex covering approximately 800 square kilometers of southwest South Carolina. SRS operations have included or presently include nuclear reactors, waste-vitrification facilities, chemical reprocessing plants, and fuel fabrication. SRS relies on the predictive capabilities of dispersion models to assess possible emergency response actions. Accurate and timely wind field input to these models is crucial. For the initial and lateral boundary conditions, steady-state (diagnostic) winds are commonly used because they can be obtained with minimal computational effort; however, their use can cause serious prediction errors during changing weather conditions. Recent advances in computing speed enables mesoscale numerical models to generate three-dimensional prognostic fields in an attempt to describe these variations. It is important to assess the accuracy of the model simulations. This paper describes how statistical measures were used to compare several observed and predicted meteorological variables for the southeastern United States spanning two full years. The period examined is from April 1998 through March 2000. Six different locations within the model domain were selected, with comparisons being made both at the surface and aloft. The majority of the operational work performed at the SRS is at or near the ground level. Therefore, much of this report focuses on comparisons between observations and simulations at the surface.

Use of mesoscale models to provide forecast results for local and regional applications are becoming widely accepted. Other applications include the work of McQueen et al. (1995), who performed model simulations for the northeastern United States and Manobianco et al. (1996), who used a model to provide forecasts for the Kennedy Space Center in Cape Canaveral. In addition, Snook et al. (1997) described the use of a mesoscale model in support of the 1996 Summer Olympic Games in Atlanta. Model verification studies include the work of Keyser and Anthes (1977), who developed a method of determining the skill of a forecast. Wilmott et al. (1985) expanded upon this technique and developed an index of agreement between observations and simulations.

These simulations were performed using the Regional Atmospheric Modeling System (RAMS, Pielke et al., 1992), a finite-difference, primitive equation, three-dimensional mesoscale model originally developed at Colorado State University. A domain with 20-km horizontal grid spacing was used to generate 24-hr forecasts of meteorological conditions over the two-state region covering Georgia and South Carolina. Since the primary use of these data at the SRS is for emergency response, the interest here will focus on winds (i.e. direction, speed, and turbulence intensity).

There are numerous difficulties in making direct comparisons of model output with observations (Nappo et al. 1982). The first difficulty involves comparing spatially averaged model results (over a grid volume) with observations representing point values including spatial and temporal atmospheric effects not necessarily accounted for in the model (i.e. sub-grid scale effects). Another problem arises from inadequate model formulation, including the omission or lack of understanding of certain atmospheric processes. For example, in these simulations, although 
clouds are generated and a convective paramaterization scheme (Pielke et al. 1992) generates precipitation, explicit microphysics to simulate rainfall, snowfall, etc. are not considered. Yet another cause of difference is inaccurate initialization. In addition to three-dimensional initial conditions provided by large-scale National Oceanic and Atmospheric Administration (NOAA) models, RAMS also uses sequential six-hour intervals of these data for lateral boundary conditions. If the large-scale data are inadequate, the mesoscale simulations will suffer. Finally, errors can be introduced at various stages in the process of collecting, transmitting, and archiving the observed data. These types of errors are neglected in this study. Systematic differences (e.g. model physics errors) should affect predicted model output in a consistent way, while data errors are generally random.

The objective statistical measures for comparing model predictions and observations used in this study were taken largely from Cox et al. (1998), who compared a suite of mesoscale model predictions (including RAMS) for a variety of global locations. From Cox et al. (1998), the forecast accuracy criterion for wind direction was chosen to be $30^{\circ}$, along with a wind speed accuracy of $1 \mathrm{~m} \mathrm{~s}^{-1}$ for winds at the surface. For comparisons at higher atmospheric levels, the range was extended to $2.5 \mathrm{~m} \mathrm{~s}^{-1}$. (Note that Cox et al. (1998) desired a range of $1 \mathrm{~m} \mathrm{~s}^{-1}$ for speeds less than $10 \mathrm{~m} \mathrm{~s}^{-1}$, while a more relaxed range of $2.5 \mathrm{~m} \mathrm{~s}^{-1}$ was desired for speeds greater than this. Such a distinction was not made here, except with regard to measurement level above ground). In addition, the preferred temperature accuracy was $2^{\circ} \mathrm{C}$.

Another prediction variable that may be examined is atmospheric turbulence intensity via the standard deviation of azimuth and elevation angle $\left(\sigma_{A}\right.$ and $\left.\sigma_{E}\right)$. These are not typically measured at National Weather Service (NWS) stations; however, the instrumented tower system at the SRS routinely makes such measurements. Properties of atmospheric turbulence intensity at the SRS were studied by Weber et al., (1975). For the current study, the RAMS code was modified to calculate $\sigma_{A}$ and $\sigma_{E}$ values at one-hour intervals, and comparisons were made with measured values. The desired range for the azimuth angle deviation was assumed to be $5^{\circ}$. This value was chosen since these values do not typically exceed $30^{\circ}$ during one-hour time periods, which is one-sixth of the horizontal wind direction range $\left(180^{\circ}\right)$, and $5^{\circ}$ is one-sixth of the horizontal wind direction criterion. The elevation angle deviation was roughly half the azimuth angle deviation, so the range for $\sigma_{E}$ deviation was assumed to be $2.5^{\circ}$. Note that the simulated values for the SRS location were only available beginning in February 1999.

The next section discusses model background, observation locations, as well as the statistical measures that were used. Statistical results are then described. The results are broken up into two major categories. The first part of the comparison deals with the aggregate 2-year period of data (or $1^{+}$year of data for SRS) in the form of scatter plots and histograms. A more detailed analysis of model performance and skill indicators as a function of both forecast time and the time of the year (i.e. monthly totals or averages) is given in the second part of the comparison. The results are briefly summarized in Section 3, while a more detailed discussion with implications is given in Section 4. Concluding remarks concerning possible reasons for the given results, as well as paths for future work are then discussed. 


\section{BACKGROUND}

\subsection{Prognostic Model (RAMS)}

The RAMS model is capable of simulating a wide range of atmospheric motions due to the use of a nested grid system. Incorporation of topographic features occurs through the use of a terrain-following vertical coordinate system, while turbulence is parameterized using Mellor and Yamada's level 2.5 scheme (Mellor and Yamada, 1982), as modified by Helfand and Labraga (1988) for growing turbulence.

Large-scale data are available from a variety of sources, including the National Oceanic and Atmospheric Administration (NOAA) and the Fleet Numerical Meteorology and Oceanography Center (FNMOC). These data are ingested at SRS from both a commercial vendor and from the internet. These large-scale data are used to generate initialization files in RAMS containing the three-dimensional large-scale observational data (horizontal velocity components, potential temperature, pressure, and moisture) interpolated to the RAMS (polar-stereographic) model grid. This interpolation was performed on isentropic and terrain-following coordinate surfaces (Pielke et. al., 1992). The initialization file corresponding to the starting time in the simulation was then used to create an initial condition for the entire three-dimensional RAMS model grid. Subsequent files (i.e. 6-hr forecasts, 12-hr forecasts, etc.) were used for lateral boundary conditions using linear interpolation in time, based on the Davies relaxation assumption (Davies, 1976).

The actual simulation covered a span of 30 hours, but the first 6 hours were purposely set aside while the model was 'spinning up' a realistic boundary layer. Although a longer 'spinup' time (i.e. 12 hours) is preferred, the limitations of the computer system at this time combined with the need for a timely forecast required using the six hour 'spinup' period. Simulations were nominally generated twice daily using analyzed dynamic meteorological fields generated by NOAA's National Centers for Environmental Prediction (NCEP) large-scale models (valid at 00 and 12 Greenwich Mean Time [GMT]). The large-scale Eta model (Mesinger et al., 1988, and Black, 1994) was used if available, while the Aviation model (based upon the Medium Range Forecast [MRF], [Sela, 1980]) was used as a backup in the event the Eta information was not received. The lowest model level was $\sim 26$ meters above ground level (AGL). Because the large-scale data typically take 3 to 4 hours to be processed at NCEP, the 6-hr forecast from the NCEP simulations were used as the initial condition for the RAMS simulations (i.e. starting at 06 or 18 GMT). Therefore, a RAMS simulation using NCEP analysis data valid at 00 GMT provided a $24-\mathrm{hr}$ forecast beginning at 12 GMT.

A soil model developed by McCumber and Pielke (1981) and modified by Tremback and Kessler (1985) was used to determine surface temperatures from surface energy balances involving net radiation, turbulent latent and sensible heat flux, and soil heat flux. Sandy clay loam soil was assumed for the southeast United States with initial soil moisture between 20 and 50\%. The 
Biosphere Atmosphere Transfer Scheme (BATS, Dickinson et al., 1986) was used for the vegetation parameterization, which further served to modify surface fluxes. Variable fractional land coverage and sea-surface temperatures were also used for input to the model.

For the two-year time span, a total of 1462 simulation periods could have been generated (two simulations per day). However, due to a variety of problems including missing large-scale data, errors in scripts used to generate the forecasts, and network or computer downtime, only 1337 simulations $(91 \%)$ were created.

\subsection{Modifications to RAMS}

Applications of the RAMS model results to the operational atmospheric transport codes used at SRS required modifications to generate standard deviations of azimuth and elevation angle $\left(\sigma_{A}, \sigma_{E}\right)$. These quantities are utilized in the dispersion calculations (e.g. see Pasquill, 1983) within emergency response codes used at SRS. The horizontal grid spacing $(20 \mathrm{~km})$ is too coarse to capture all turbulent activity. Thus, the subgrid-scale parameterization (Mellor and Yamada, 1982) scheme must be used to determine turbulent moments. The atmospheric turbulence intensities are comprised of a resolved and subgrid portion

$$
\sigma_{A}=\sigma_{A_{R}}+\sigma_{A_{S}}
$$

where the resolved portion accounts for deviations over a user-specified time at the resolvable scale (in this case, over the past hour). Ignoring cross correlations, the deviations are calculated for the horizontal plane ( $u$ denotes the zonal component, while $v$ denotes the meridional component) from:

$$
\sigma_{A_{R}}=\frac{\sqrt{\sigma_{u}^{2}+\sigma_{v}^{2}}}{\bar{s}}
$$

where the variance terms are expressed as:

$$
\sigma_{u}^{2}=\overline{u^{\prime} u^{\prime}}=\frac{1}{N-1} \sum_{i=1}^{N}\left(u_{i}-\bar{u}\right)^{2},(\text { for the } u \text {-component, similarly for } v \text { and } w \text { ). }
$$

Mean quantities denoted with overbars are calculated as:

$$
\bar{u}=\frac{1}{N} \sum_{i=1}^{N} u_{i}
$$

for each individual timestep, $i$. The total averaging period accesses $N$ timesteps. Note that the mean wind speed is obtained from the vector components $u$ and $v$ : 


$$
\bar{s}=\frac{1}{N} \sum_{i=1}^{N} \sqrt{u_{i}^{2}+v_{i}^{2}}
$$

For the subgrid terms, the turbulence parameterization provides $\sigma_{u}^{2}$ and $\sigma_{v}^{2}$ directly. Typically, the resolved components are negligible compared with the subgrid terms for a grid spacing greater than $10 \mathrm{~km}$. The standard deviation of elevation angle is similarly calculated with vertical velocity in the numerator:

$$
\sigma_{E_{R}} \cong \frac{\sqrt{\sigma_{w}^{2}}}{\bar{s}}
$$

and

$$
\sigma_{w}^{2}=\overline{w^{\prime} w^{\prime}}=\frac{1}{N-1} \sum_{i=1}^{N}\left(w_{i}-\bar{w}\right)^{2}
$$

\subsection{Observations}

The observed surface values were taken from NWS stations (available at 1-hr increments) and from the SRS towers. The first source was from the Meteorological Airways Format (METAR) surface station reports, available every hour. Measurements of wind speed were taken at 10 meters AGL, while temperature measurements were recorded at roughly $2 \mathrm{~m}$ AGL. It is important to note that the lowest model level in the RAMS simulations was $26 \mathrm{~m}$ AGL, which differs from the measurements at the National Weather Service sites ( 2 to $10 \mathrm{~m}$ ) leading to differences in results. Typically, observations at lower levels will yield lower wind speeds and temperatures. Of course, when an inversion exists, the temperatures will be higher.

It has been observed by SRS meteorologists and others that values for surface wind speeds as generated by the NCEP-Eta model tend to be biased high in the southeast United States. Since this large-scale model is used to generate initial and lateral boundary conditions within RAMS, a roughness length parameter $\left(z_{0}\right)$ used to control surface fluxes of momentum, heat, and moisture (Thom, 1975) was increased to 0.50 meters. (A value of $z_{0}=0.01 \mathrm{~m}$ represents a smooth surface and $z_{0}=1.0 \mathrm{~m}$ indicates a very rough surface, such as a forested area). The increase in $z_{0}$ creates more friction in the lower model levels, effectively lowering wind speeds and increasing turbulence intensity. To a lesser degree, the vertical temperature profile is also affected (e.g. Panofsky and Dutton, 1984). A sensitivity study of simulated surface wind speeds to $z_{0}$ was conducted for six successive days during the first week of April 1998. Using 00 GMT largescale Eta data to initialize RAMS, four simulations (creating a 24-hour forecast period) were created for each day changing only the value for $z_{0}$. For the domain (centered on the SRS, and used in the remainder of this study), 45 METAR surface stations (reporting hourly) were selected and simulated results at the surface were computed at the station locations. Results as averaged from all reporting stations over all times of day, as well as over the 6 days are shown in Table 1. The computed mean differences and mean absolute differences (simulated less observed, as discussed in detail in the next section) show overestimates in surface speeds for smaller values of 
$z_{0}$, and a slight underestimate in simulated speeds with $z_{0}=0.50 \mathrm{~m}$. The overall mean absolute difference decreases with increasing $z_{0}$. Although somewhat arbitrary, the value of $0.5 \mathrm{~m}$ was a good compromise based on this sensitivity study, and used in the input for these simulations.

Unfortunately, surface flux information from the 1337 simulations was not archived. Therefore, using similarity theory (e.g. Dyer and Hicks 1970, Wilson 2001) to estimate simulated results at $10 \mathrm{~m}$ (and 2-m AGL) was not possible. Conversely, the process of estimating observations at a higher level contained too many unknown parameters (including variable surface conditions among the various NWS stations) to warrant this added degree of complexity in the calculations. Therefore, comparisons were made between the lowest model surface layer and observations with the understanding that values have been affected in part by the increased roughness length assumption.

The other surface data source was the climatology tower at the SRS, which gives information at several levels every 15 minutes (Kurzeja, 1993). Since observations at the tower can be obtained at both 18 and $36 \mathrm{~m}$ AGL, an interpolated value between these levels to the RAMS 26-m level was calculated. Values of turbulence intensity $\left(\sigma_{A}, \sigma_{E}\right)$ were also compared at SRS. Unfortunately, the simulated data interpolated to this SRS location were only available beginning in February 1999. Observations at higher levels (fixed pressure levels) were obtained from upper-air data available at 00 and 12 GMT.

For the statistical comparisons, surface data were collected at 5 different locations throughout the modeling region (Fig. 1). The stations were chosen due partly to the reliability of the station in reporting data, and partly for the geographical location. Atlanta, Georgia was chosen as a westerly location, while Alma, Georgia denoted a location in the southern portion of the domain. Measurements from Bush Field in Augusta, Georgia were chosen because it is close to the SRS, while Charleston, South Carolina allowed for a coastal environment to be analyzed. Finally, Charlotte, North Carolina was selected due to its proximity to the Appalachian Mountains, as well as its northern location within the modeling domain. The upper-air data were available only at Atlanta and Charleston.

\subsection{Statistical Comparisons}

For each of the six different locations discussed in the introduction, three separate meteorological variables were examined: wind direction, wind speed, and temperature. In addition, for the SRS location, $\sigma_{A}$ and $\sigma_{E}$ were considered. Differences in surface values were first investigated. For each of these variables, simulated and observed surface data were compared at RAMS forecast times of $0,6,12,18$, and 24 hours. The wind speed, turbulence intensities, and temperature were compared using scatter plots. Generic statistical values were also calculated for these comparisons. The mean difference between simulation and observation is given by

$$
\bar{d}=\frac{1}{N} \sum_{i=1}^{N}\left(s_{i}-o_{i}\right)
$$


where $N$ is the total number of points to compare (in which both the observed and simulated values exist), $s_{i}$ is the simulated value of the $i^{\text {th }}$ point, and $o_{i}$ is the observed value of the $i^{\text {th }}$ point. Similarly, the mean absolute bias was determined by taking the absolute difference between all simulated and observed values and determining an average

$$
\overline{|d|}=\frac{1}{N} \sum_{i=1}^{N}\left|s_{i}-o_{i}\right|
$$

Finally, the standard deviation of the difference is given by:

$$
\sigma_{\text {Diff }}=\left(\frac{1}{N-1} \sum_{i=1}^{N}\left[\left(s_{i}-o_{i}\right)-\bar{d}\right]^{2}\right)^{1 / 2}
$$

The percentage of data points falling within the desired criteria were also determined. Sampling distributions for the means are typically assumed to be normal if $N$ is large.

The wind direction was handled differently due to the problems associated with overlap from $0^{\circ}$ and $360^{\circ}$. (For example, the difference for $\theta_{\mathrm{s}}=350^{\circ}$ and $\theta_{\mathrm{o}}=10^{\circ}$ may be interpreted as $340^{\circ}$ or as $20^{\circ}$ ). Thus, wind direction differences were constrained to be an absolute value between $0^{\circ}$ and $180^{\circ}$. A histogram of absolute differences in wind direction was generated for the different forecast times and locations.

A comprehensive technique of evaluating the 'skill' of the model simulations uses the rootmean-square error (RMSE), the ratio of RMSE before and after removing a bias, the standard deviation, and an index of agreement. This method is described in Willmott et al. (1985) and used by others (e.g. Steyn and McKendry, 1988, and Ulrickson and Mass, 1990). Some statistical definitions and discussion are necessary.

The root mean square error may be written:

$$
R M S E=\left[\frac{\sum_{i=1}^{N} w_{i}\left|\mathbf{s}_{i}-\mathbf{o}_{i}\right|^{2}}{\sum_{i=1}^{N} w_{i}}\right]^{1 / 2}
$$

where for $N$ comparisons, the $i^{\text {th }}$ part is due to a simulated value $\mathbf{s}_{i}$ and an observed value $\mathbf{o}_{i}$. Note that s (and o) may be a vector (i.e. horizontal wind speed components) or scalar quantity (temperature). For vector quantities, the magnitude of the vector differences must be used in the determination of the difference. The term $w_{i}$ is a scalar weight accounting for spatial or temporal irregularities in the comparison fields. In practice, $w=1.0$ for all $i$ in this study, since the time 
interval over which our calculations are made is constant. Biases may exist in the RMSE (due to a variety of factors, including incorrect boundary condition specification). One type of bias may be removed using the following (Keyser and Anthes, 1977):

$$
R M S E C=\left[\frac{\sum_{i=1}^{N} w_{i}\left|\left(\mathbf{s}_{i}-\overline{\mathbf{s}}\right)-\left(\mathbf{o}_{i}-\overline{\mathbf{o}}\right)\right|^{2}}{\sum_{i=1}^{N} w_{i}}\right]^{1 / 2}
$$

where $\overline{\mathbf{s}}$ and $\overline{\mathbf{o}}$ are the mean values of $\mathbf{s}$ and $\mathbf{o}$ over the $N$ datapoints. One can define the ratio of "unbiased" to biased RMSE as (Pielke and Mahrer, 1978):

$$
R_{U B}=\frac{R M S E C}{R M S E}, 0 \leq R_{U B} \leq 1
$$

Values of $R_{U B}$ approaching unity indicate the numerator and denominator are approximately the same size which indicates either the bias approaches 0 or the variance is very large compared to the bias. On the other hand, if $R_{U B}$ approaches 0 the RMSE is much larger than the RMSEC, indicating a significant bias. From Eq. (13), when RMSEC equals RMSE, either the bias equals zero, or the variance equals zero. For zero bias, $R_{U B}=1$. When the variance is zero, the bias also equals zero, rendering Eqn. (13) indeterminate. However, the latter case would never occur in a practical sense since this only occurs when every single simulated value equals the observed value, so the desired value is unity in this study.

If the standard deviation of both simulation and observation is calculated:

$$
\begin{aligned}
& \sigma_{S}=\left[\frac{\sum_{i=1}^{N}\left(\mathbf{s}_{i}-\overline{\mathbf{s}}\right)^{2}}{N-1}\right]^{1 / 2} \\
& \sigma_{O}=\left[\frac{\sum_{i=1}^{N}\left(\mathbf{o}_{i}-\overline{\mathbf{o}}\right)^{2}}{N-1}\right]^{1 / 2}
\end{aligned}
$$

then the following ratios may be determined:

$$
R_{R M S}=\frac{R M S E C}{\sigma_{o}},
$$




$$
R_{S T D}=\frac{\sigma_{S}}{\sigma_{O}} .
$$

According to Keyser and Anthes (1977), $R_{R M S}$ should be less than unity to show skill in a prediction, while $R_{S T D}$ should be near unity for realistic predictions. It is important to note that Eqns. (16) and (17) are not independent. The results for $R_{R M S}$ are different depending on if $R_{S T D}$ is greater or less than unity. A higher linear correlation is required if $R_{S T D}>1$. For example,

although $R_{S T D}=2$ and $R_{S T D}=1 / 2$ may have the same correlation, the conclusions about $R_{R M S}$ may be different. Because the restrictions on the correlation are tighter for the former case, it is better for $R_{S T D}$ to be less than unity.

An index of agreement is also discussed in Willmott et al. (1985):

$$
I=1-\frac{\sum_{i=1}^{N} w_{i}\left|\mathbf{s}_{i}-\mathbf{o}_{i}\right|^{2}}{\sum_{i=1}^{N} w_{i}\left(\left|\mathbf{s}_{i}-\overline{\mathbf{o}}\right|+\left|\mathbf{o}_{i}-\overline{\mathbf{o}}\right|\right)^{2}}
$$

which is unitless and ranges between 0 (no agreement between simulation and observation) and 1 (perfect agreement). It is a measure of the model differences from the mean observation as compared with the observed differences from the mean observation.

For these skill comparisons, we made statistical comparisons of the winds (speed and direction combined) and temperature. For the wind vector, the $u$ and $v$ components were again considered. Therefore, the magnitudes were written

$$
\begin{aligned}
& \mathbf{s}_{i}=\left[u_{s, i}^{2}+v_{s, i}^{2}\right]^{1 / 2} \\
& \mathbf{o}_{i}=\left[u_{o, i}^{2}+v_{o, i}^{2}\right]^{1 / 2}
\end{aligned}
$$

while for the temperature comparisons

$$
\begin{gathered}
s_{i}=T_{s, i} \\
o_{i}=T_{o, i}
\end{gathered}
$$

were used for simulated and observed quantities, respectively. 


\section{RESULTS}

\subsection{Ensemble Averages}

An 'ensemble' plot considering all of the six different locations, was first examined for wind direction, wind speed, and temperature. The same type plot for $\sigma_{A}$ and $\sigma_{E}$ using SRS data alone was also investigated. As previously discussed, horizontal wind direction differences were constrained to be an absolute value between 0 and $180^{\circ}$. Furthermore, the differences were divided into bins of $15^{\circ}$ each. Histogram plots (Fig. 2) are shown for each forecast time for the ensemble of five regional city locations as well as the SRS, indicating the percentage of times that the error in wind direction fell within a certain range. Note that the final bin contains all differences between $90^{\circ}$ and $180^{\circ}$. The absolute mean bias for all compared data (Eqn. 9) was also calculated and is shown as a dashed vertical line in Fig. 2. Note that the total number of data points $N$ (times in which both observation and simulation exist, No.Pts.) for each forecast time is also indicated. A summary of statistical results for each individual location is given in Table 2. There is a tendency for directional errors to increase with forecast time.

Horizontal wind speed values are important in emergency response to determine plume dilution. Scatter plots for wind speed $\left(\mathrm{m} \mathrm{s}^{-1}\right)$ are shown in Fig. 3 for the ensemble, with a desired accuracy range of $\pm 1 \mathrm{~m} \mathrm{~s}^{-1}$ (given by the dotted lines bracketing the $45^{\circ}$ line). Observed values are displayed along the $x$-axis and simulated values along the $y$-axis. The total number of comparisons for each forecast time, $N$, is used to determine a mean difference $\bar{d}$ (Mean), mean absolute difference $\overline{|d|}$ (AbsBias), and standard deviation $\sigma_{\text {Diff }}$ (St.Dev.) as given by Eqns. (8) to (10). The discrete nature of the scatter points results from the limited number of significant digits available from the METAR reports and the small range over which the data are plotted. (Note that this is not a problem with the SRS tower data, where wind speed is reported to the nearest tenth of a meter per second). Tabular statistics for all locations are given in Table 3 . The overall mean difference at all times for the ensemble of locations is slightly positive, which is not surprising considering the difference in measurement height between the observations and simulations.

The standard deviation in azimuth angle and elevation angle represents the atmospheric turbulence intensity that is critical in determining pollutant dispersion. The scatter plots given in Figs. 4 and 5 for $\sigma_{A}$ and $\sigma_{E}$, respectively, contain only observations made at the SRS from February 1999 onward. Azimuth angle values tend to cluster around $15^{\circ}$ (Fig. 4) while $\sigma_{E}$ values are most often between $5^{\circ}$ and $10^{\circ}$ (Fig. 5).

Scatter plots of temperature $\left({ }^{\circ} \mathrm{K}\right)$ are shown in Fig. 6 for a bracketing range of $2^{\circ} \mathrm{K}$, while Table 4 gives information for each individual location. A glaring bias is not readily apparent from the scatter plots, although temperatures less than $\sim 0^{\circ} \mathrm{C}$ tend to be underestimated. With the exception of the 18-hr forecast period, the overall tendency is for temperature predictions to be slightly high. 


\subsection{Time Series of Statistics}

A plot of average absolute bias as a function of forecast time for Augusta is depicted in Fig. 7 for winds and temperature. This figure contains differences between observations and simulations at 2-hr intervals (from the 0-hr to the 24-hr time period) as averaged over an entire month for simulations using 00 GMT large-scale analysis times (with forecast times beginning at 12 GMT). Similar results may also be obtained using the 12 GMT large-scale analysis time simulations (with forecasts beginning at 00 GMT the following day), but are not shown. The three plots indicate comparisons of different meteorological variables. Each line within the plot represents a different month (covering the 2-yr period of interest). Once again, the wind direction bias is forced to be between $0^{\circ}$ and $180^{\circ}$. Plots of this kind can be generated for each city. However, the major points may be concluded from the results for Augusta. These figures are useful because conclusions may be determined regarding forecast accuracy as a function of the time of year, as well as the time of day. Of the three variables, only temperature clearly exhibits trends that hold for different seasons of the year.

The surface conditions may also be examined by taking the mean value over an entire month $(x$ axis) for each location and plotting the differences ( $y$-axis). In this instance, forecast times of 0 , 12, and 24 hours are investigated. The wind direction comparisons (Fig. 8) are again constrained to absolute values between $0^{\circ}$ and $180^{\circ}$. In addition, a line is shown indicating the "total" absolute bias as averaged over the entire period for all locations. The best predictions typically occur during the cooler winter months, and are worst during the warmer months. Note also that the accuracy declines with increasing forecast time ( $\sim 15 \%$ from 0 to 24 hours).

For wind speed (Fig. 9) both the mean absolute and mean relative differences are calculated for each month for these forecast periods. Two columns are used, with the left-hand column illustrating the absolute difference for each month and location over the two-year period. The right-hand column shows mean relative differences to indicate over and under-estimates as a function of the time of year. As a means of comparison, similar plots using the surface data shown previously in the scatter plots are generated showing comparisons for the 5 cities at the surface (using the METAR observations), and the SRS location (from February 1999 onward). Wind speed bias at the surface is typically within $1 \mathrm{~m} \mathrm{~s}^{-1}$, and trends as a function of the time of year are difficult to detect.

Time-dependent plots of azimuth and elevation angle deviations (in similar format to Fig. 9) are shown in Fig. 10 for the SRS location. The decline in predictive capability with increasing forecast time is not seen for these variables, as 'total' absolute differences over all months remains nearly constant. For all forecast times, there is a trend toward better agreement for $\sigma_{E}$ over the last half of the comparison period (i.e. September 1999 to March 2000), as compared to the first half of the period. The tendency for over-prediction in $\sigma_{A}$ and under-prediction in $\sigma_{E}$ as seen in Figs. 4 and 5 is also illustrated in the relative difference column.

Comparisons of surface temperature differences are illustrated in Fig. 11. The positive bias in simulated temperatures is clearly seen (and indicated in Fig. 6). The mean relative difference is 
positive in a majority of locations and months of the year. Results are also seen to degrade slightly with longer forecast times, as illustrated by the averaged mean absolute difference over all locations and times.

\subsection{Upper-Air}

Tables similar to those showing surface results (Tables 2 to 4) are given for the upper-air comparisons in Tables 5 to 7 for wind direction, wind speed, and temperature, respectively, at four different pressure levels $(850,700,500$, and 300 mbar) for Atlanta and Charleston. In this instance, the desired wind speed range criterion is increased to $\pm 2.5 \mathrm{~m} \mathrm{~s}^{-1}$ because the speeds are so much larger aloft.

Time series plots similar to Figs. 8, 9 and 11 are given in Figs. 12 to 14 for the upper-air comparisons showing differences as averaged over different months throughout the two-year comparison period. For brevity, each variable is examined at only one of the pressure levels.

In general, wind direction predictions become less accurate with increasing forecast time but better with height (Table 5). For the 700-mbar pressure level plots shown in Fig. 12, it is clear that directional error is highest during the warmer months of the year. On the other hand, wind speeds are better predicted near the surface (compare Fig. 9 with the differences at the 500-mbar level shown in Fig. 13). The tendency here is for wind speeds to be underestimated at most upper levels (Table 6).

Temperature differences at higher levels in the atmosphere are much less than at the surface (compare Tables 4 and 7), with the $2^{\circ} \mathrm{C}$ criterion being realized roughly $90 \%$ of the time. Timeseries plots at the 300-mbar level (Fig. 14) reveal a tendency for RAMS to overestimate the temperatures. There is only a slight degradation in forecast accuracy over the 24-hr period.

\subsection{Skill}

Skill comparisons are used to further examine the simulations. The RMS ratio given in Eq. (16) is illustrated in Figs. 15 and 16 for the 6 locations previously discussed. In Fig. 15, average values over all months at a given forecast time are shown for vector winds (top panels) and temperature (bottom panels). In addition, the comparison is broken up into 00 GMT (i.e. 0-hr forecast corresponding to 12 GMT, or mid-morning) and 12 GMT simulations. Values tend to be closer to unity for the 12 GMT simulations, indicating less skill. Figure 16 shows $R_{R M S}$ as a function of month for each location. While winds do not show a pattern, the skill in predicting temperature seems to worsen during the summer months.

Similar plots illustrating the ratio of "unbiased" to biased RMS error (Eqn. 13), $R_{U B}$, are shown in Figs. 17 and 18. It is clear that a more significant bias exists for temperature than for winds. A forecast-time dependence is not exhibited for winds, but clearly for temperature at all locations except the SRS. Monthly variations in bias (Fig. 18) do not exhibit a pattern. Similar illustrations of the ratio of simulated-to-observed standard deviation $\left(R_{S T D}\right.$, Eqn. (17)) are shown 
in Figs. 19 and 20. For winds, the clearest problem is again at Alma. Seasonal variations for winds are not evident, but a clear dependence for temperature is noted.

Finally, Figs. 21 and 22 show plots of the index of agreement (Eqn. 18). As expected from the previous skill results, the index is lower for temperature, especially during warmer months (Fig. 22). In addition, the index tends to be worse for the 12 GMT simulations than for the 00 GMT simulations.

\section{DISCUSSIONS}

\section{(a) Ensemble averages}

It is evident that the absolute mean bias for wind direction (Fig. 2) increases with forecast time when considering the ensemble. The best agreement typically occurs at the 0-hr forecast period. Atlanta, Charleston, and the SRS showed a trend of increasing absolute bias over time, while Augusta showed an increasing trend from 0 to 18 hours with a slight decrease for 24 hours. Alma and Charlotte results were ambiguous. The best forecasts occurred at Alma for 0, 12, and 24 hours and at Charleston for 6 and 18 hours, while the worst forecasts occurred at Charlotte for 0,12 , and 24 hours and at Augusta for 6 and 18 hours. The absolute mean bias generally ranges from 30 to 40 degrees for all of these locations and at all times. The absence of nocturnal measurements at Alma leads to improvements in direction comparisons, due to the absence of light and variable conditions typically present at night. Absolute wind direction errors less than $15^{\circ}$ occur roughly a third of all times, while errors less then $30^{\circ}$ are registered 55 to $60 \%$ of the time. This latter figure compares favorably with values obtained by Cox et al. (1998) when using RAMS. (Note that Cox et al. (1998) use a horizontal grid spacing of 40-km, which would imply that results from the current simulations should be better). On the other hand, extremely poor forecast wind direction $\left(>75^{\circ}\right)$ occurs between 10 and $15 \%$ depending on the location. For emergency response use, it is important that directional errors of this kind be reduced.

The scatter plots for wind speed (Fig. 3) do not indicate a trend in bias with respect to forecast time. However, there is a tendency for observed speeds greater than $8 \mathrm{~m} \mathrm{~s}^{-1}$ to be underestimated by the model. Examining mean differences for each time and location (Table 3), it is evident that wind speeds at Atlanta are underestimated, while speeds at Augusta, Alma, and SRS are overestimated. The absolute mean biases range from 1.0 to $1.6 \mathrm{~m} \mathrm{~s}^{-1}$. The standard deviation for differences are on the order of $1.5 \mathrm{~m} \mathrm{~s}^{-1}$. The predicted wind speed was within $1 \mathrm{~m} \mathrm{~s}^{-1}$ of the observations between 60 and $84 \%$ of the time, with the best performance at the SRS ( 80\%), followed by Augusta, Charleston, and Charlotte (>70\%). Cox et al. (1998) obtain roughly 50 to $70 \%$ compliance, with a larger reduction in performance at the 12 and 24-hr forecast periods.

The performance at the 6 and 18-hr forecast times is worse than the 0,12 , or 24-hr periods for Augusta, Alma, and the SRS, while the discrepancy is not so severe at the other locations. This can be traced to the average observed wind speed over the entire period, which is 10 to $30 \%$ higher at the 6 and 18-hr forecast times (corresponding to higher wind speeds during the midafternoon time period). Thus, the potential for differences in simulated and observed values is 
increased. For Augusta and Alma in particular, the relative difference in speeds between these forecast times is greater $(\sim 30 \%)$ than for the other locations $(\sim 10 \%)$. Therefore, the forecast is worse relative to the other times. The number of comparisons of winds is significantly less than temperature for Alma and Augusta in particular (compare $N$ in Tables 2 and 3, with $N$ in Table 4). If a wind is reported as calm within the observed report, then a comparison of wind speed and direction is not made. For these two locations, a much higher percentage of calm winds were reported. Observed wind speed is limited by the resolution of the measurement. Anything quite small (but non-zero) is recorded as "calm", which is not considered in the comparisons. Assuming the predicted values at these times will also generally be lower, then both the average predicted and observed values for wind speed after removal of these data points is higher than if they were included. Of course, this does not mean that the difference will drop if simulated speeds during this time remain high. Another reason for Alma's poor performance is the absence of measurements during the nocturnal period when wind speeds are typically lower. (Again, this implicitly assumes wind speed differences tend to be higher for periods with larger absolute noncalm wind speeds).

It is difficult to assess the 'value' of the turbulent intensity comparisons. However, several items may be noted. For the azimuth angle (Fig. 4), the desired range is met 64 to $77 \%$ of all times. Agreement is worse for the 6 and 18-hr forecast periods, with a tendency for RAMS to underpredict the value of $\sigma_{A}$. These times represent the mid-afternoon and early-morning. During the nocturnal period ( 01:00 LST), this probably indicates that RAMS is simulating a more stable atmosphere than is measured. The standard deviation between measurements and simulations is also significantly higher during these time periods. On the other hand, the mean bias is positive for forecast times of 0,12 , and 24 hours.

The deviation in elevation angle (Fig. 5) is underestimated at all times, with worse comparisons (i.e. higher absolute bias and lower percentage of data within the $2.5^{\circ}$ bracket) again evident at the 6 and 18-hr forecast times. The standard deviation and absolute mean bias values are all roughly half the values for the azimuth angle deviations. However, RAMS tends to severely over-predict $\sigma_{E}$ for a few instances (especially for the 6 and 18-hr forecast times), with much smaller under-predictions at most other times. The threshold of $2.5^{\circ}$ is obtained with nearly the same frequency as $\sigma_{A}$. The reason for the underestimates in $\sigma_{E}$ is related to the vertical velocity (Eqn. (7)). Previous studies using RAMS (Lyons et al., 1995) have shown finer horizontal grid spacing results in larger vertical velocity terms. The variation in vertical velocity for $20-\mathrm{km}$ grid spacing is not as great as the measured values, leading to an under-prediction in the elevation angle deviation. It is also clear from these plots that the turbulence estimates within RAMS are not as reliable as the horizontal wind estimates. This is likely due to errors generated using the turbulence parameterization scheme (Mellor and Yamada, 1982). In particular, the choice of length scales has been shown to affect the amount of turbulence generated by the model (Lascer and Arya, 1986).

The ensemble of temperature comparisons reveals (Fig. 6, Table 4) that although values of absolute mean bias in temperature $\overline{|d|}$ at the earliest time tend to be closer to observations than at 
the later times, a strictly increasing difference is seen only at Charlotte and the SRS. The percentage of predictions that are within $2^{\circ} \mathrm{K}$ of the measurement varies from $48 \%$ to $75 \%$, with the best results at Charleston and the SRS, and the worst at Augusta. This also is slightly better than the results documented (40 to 60\%) in Cox et al. (1998). In Table 4, the mean difference in simulated and observed temperature indicate a tendency for over-prediction at nearly all of these forecast times. However, generally lower values are seen at the 6 and 18-hr forecast periods. The largest over-predictions occur at Augusta, possibly a result of the geographical location of Bush Field within a river valley. Note that the SRS results (observations taken at $26 \mathrm{~m}$ AGL) are not distinctly better when compared with the other regional city locations (observations at $2 \mathrm{~m}$ AGL), as was the case for wind direction and speed. Although the height at which the measurements are taken should influence the comparisons, a great difference is not seen here for temperature.

\section{(b) Time Series of Statistics}

The time-series plots (Figs. 7 to 11) reveal several interesting features. The wind direction error as a function of time of day (Fig. 7a) tends to be worst after the transition period from sunlight to darkness ( $\sim 0$ GMT to 06 GMT). However, this does not seem to be the case for wind speed (Fig. 7b). The over-predictions in surface wind-speed as seen in the mean differences at Alma (Table 3) and the under-predictions at Atlanta (Fig. 9), are clearly seen during most months in the relative difference column. Again, differences between prediction and observation rise slightly with increasing forecast periods. Finally, the decrease in mean difference for temperature seen in Fig. 6 and Table 4 is due to values of temperature in the model tending to be underpredicted during the daytime hours between 12 and 21 GMT, and over-predicted between 00 and 12 GMT (Fig. 7c). Thus, in this instance a forecast time of 6 hours would be at 18 GMT. For the Alma case in particular, the difference is magnified because observed data are not available between 04 and 08 GMT, a nocturnal period when RAMS tends to over-estimate temperatures.

The following points may be made regarding time-dependent errors for temperature (Figs. 7 and 11, Table 4). The worst positive bias typically occurs during the warmer months, except at Charleston and Alma, when maximum differences occur during late autumn. The worst negative bias typically occurs during the cooler months. There is a large shift in bias from under- to overprediction at $\sim 00$ GMT and from over- to under-prediction at $\sim 12$ GMT. This is related to the radiative transfer calculations within RAMS. There is also a clear relationship with simulated moisture content during this transition (00 GMT). On those occasions when temperature is severely under-predicted, dew-point temperature is severely over-predicted (not shown). In this case, the soil moisture content may be too large initially, leading to wetter, cooler conditions than observed. Alternatively, when the temperatures are too high, dew-point temperature is often too low. The soil moisture content may be too low initially, resulting in dryer, hotter conditions than measured.

\section{(c) Upper-air}


The absolute mean difference in wind direction (Table 5) drops from $25^{\circ}$ to $30^{\circ}$ at 850 mbar down to $10^{\circ}$ to $15^{\circ}$ at 300 mbar, resulting in a much higher percentage of predictions within the $15^{\circ}, 45^{\circ}$, and $75^{\circ}$ range than at the surface. Results are generally better in Atlanta for the different heights, while increasing forecast times leads to a reduction in the accuracy of the simulations. As a means of comparison, Cox et al. (1998) obtained upper-air percentages between 80 and $95 \%$ for a range of $30^{\circ}$ for all vertical levels (10 levels between 1000 and 100 mbar) considered. At the $30^{\circ}$ interval for the 4 levels and cities chosen in this study (not shown in Table 5), the average percentages are $\sim 80 \%$, which can also be discerned by interpolating between values for 15 and $45 \%$. The predicted wind direction is clearly poorer at the surface (Fig. 8), where averaged mean absolute differences are on the order of $35^{\circ}$. The forecasted wind direction accuracy declines from the 0 -hr to the 24-hr forecast, but improves with height above ground (Fig. 12). As seen in Table 5 for all times of the year, the latter is expected since there is less variability in direction at the lower pressure levels. In all cases and locations, wind direction error is worse during the warmer months, as previously discussed (Figs. 7 and 8).

Examination of wind speeds comparisons at the upper levels (Table 6) reveals a slight underestimate of winds at all levels and both locations, with the exception of Atlanta at 300 mbar. The absolute mean bias tends to increase with forecast times, as well as with height. The latter occurs because the magnitude of the speed at lower pressure levels is generally higher. The desired criteria are met between 50 and $80 \%$ of the time, which is somewhat lower than that obtained by Cox et al. (1998). Values for $\sigma_{\text {Diff }}$ and $\overline{|d|}$ would suggest that comparisons are slightly better at Atlanta, except at the highest vertical level (300 mbar). Comparison of surface and 500-mbar level values show that predictions near the surface (Fig. 9) are superior to values aloft. The mean absolute differences in speed increase with decreasing pressure level $\left(\sim 1.1 \mathrm{~m} \mathrm{~s}^{-1}\right.$ at the surface, $\sim 2.5 \mathrm{~m} \mathrm{~s}^{-1}$ at 850,700 , and $500 \mathrm{mbar}$, and $\sim 3.5 \mathrm{~m} \mathrm{~s}^{-1}$ at $300 \mathrm{mbar}$ ). Degradations in accuracy again occur with increasing forecast time. The increase in error with height was previously discussed, and the RAMS forecasts tend to under-predict the speeds at the three highest levels, especially at the 12 and 24-hr forecast periods. Although there is no trend at the 850 and 700-mb levels, the absolute differences tend to increase during the cooler months at the 500 and 300-mb levels.

Finally, temperature comparisons (Table 7) indicate a great improvement over those values obtained at the surface (Table 4). For all levels and both locations, the $2{ }^{\circ} \mathrm{C}$ criterion was reached between 85 and 98\%, which is comparable to that of Cox et al. (1998). Mean differences reveal overestimates at Atlanta, except at the 700-mbar pressure level, while in Charleston, underpredictions exist at all levels except 300 mbar. Nearer the surface (i.e., 850 mbar), comparisons are better at Charleston, while aloft simulations at Atlanta are better. Deviations are less than $2{ }^{\circ} \mathrm{C}$ at all levels and both locations, while absolute mean bias is a minimum at 500 mbar. Temperature differences (Fig. 14) indicate that the bias is clearly degraded near the surface (compare Figs. 11 and 14). At the 700-mb pressure level (not shown), temperatures tend to be underestimated, especially at the 24-hr forecast time, while at the $300-\mathrm{mb}$ level, temperatures are slightly overestimated. Atlanta comparisons also tend to be higher relative to Charleston as seen 
in Table 7. The best results occur during the warmer months (i.e. May, June, July), while the mean absolute differences tend to rise when it is cooler.

\section{(d) Skill}

Finally, the skill comparisons (Figs. 15 to 22) are discussed. For wind comparisons (Fig. 15), all locations show $R_{R M S} \sim 0.5$, except for Alma. Values for $R_{R M S}$ for temperature are slightly higher than for winds. Ratios for RMS at Charleston and the SRS appear to be lowest as a function of forecast hour (Fig. 15), while values at Augusta are poorest. On a monthly basis (Fig. 16), $R_{R M S}$ for winds do not generally indicate a trend as a function of the time of year, with the exception of Alma, which worsens during the warmer months. The maximum ratios for temperature are seen during the summer of 1998, and somewhat less during the warmer season of 1999. For temperature, the ratios approach unity in most locations during the late morning for the 12 GMT simulations, with a drastic decline in performance after 12 GMT (as seen in Fig. 7). It is interesting to note that a similar reduction in performance for the 00 GMT simulations (i.e. for a forecast time of two hours) is not noticed.

Ratios of unbiased-to-biased RMS error in the simulations (Fig. 17) show that for temperature, $R_{U B}$ exceeds 0.8 in most locations, except during late afternoon or early evening (forecast hour 10 to 12, compare with overestimates seen in Fig. 7c). Examination of bias removal on a monthly basis reveals the most bias in winds (Fig. 18) occurs at Alma, while the most bias in temperature occurs at Augusta. A particularly strong bias correction is evident at Augusta for both 00 and 12 GMT (Figs. 17 and 18) simulations at a time just after sunset ( 02 GMT), which most certainly coincides with Bush Field airport's location within the Savannah River valley. With 20-km horizontal grid spacing, the RAMS simulations are not able to adequately simulate this feature. The bias removal for temperature indicates little trending, with the exception of June and July 1999, where significant biases relative to the mean were seen at most locations. As indicated in Keyser and Anthes (1977), the existence of these biases could indicate incorrectly specified boundary conditions.

The ratio of simulated to observed standard deviation (Figs. 19 and 20) reveals the largest errors for Alma. This is likely because measured winds are not accounted for during the nocturnal period when winds are lighter, generally resulting in lower RMS and deviation errors. The simulated deviations of temperature are near unity over most forecast times (Fig. 19), with a decline in performance again shortly after sunrise. Again, the 12 GMT simulations generally indicate poorer performance than the 00 GMT simulations. As stated previously, it is more desirable for this ratio to be less than unity since $R_{R M S}$ can be less than unity with a smaller, but positive, correlation coefficient. Therefore, use of $R_{S T D}$ as a statistical measure implies that winds simulated at Atlanta $\left(R_{S T D}<1\right)$ are more realistic than at the SRS $\left(R_{S T D}>1\right)$. The dependence between $R_{R M S}$ and $R_{S T D}$ may also be seen by the lower $R_{R M S}$ values relative to unity (Fig. 15) for winds at Atlanta.

As a function of the time of year (Fig. 20), seasonal trends in the ratios for winds are not as obvious, although it could be argued that the Alma, GA and SRS locations tend to be higher 
during the warmer months. The temperature ratios fluctuate based on season, with values exceeding unity during the cooler months and being much less than unity during the warmer months. The reduced temporal variance in the simulated fields during the summer indicates greater uniformity in surface temperatures over time. During the cooler months, the range of predicted temperatures is larger than observed, probably due in part to large underestimates in very cold temperatures in the model (e.g. Fig. 6), which leads to the higher standard deviations. The trend for the absolute difference in temperatures also supports this assertion (Fig. 11). Again, it may be argued that temperature comparisons as a function of the time of year are better during the warmer months when $R_{S T D}<1$.

For the index of agreement wind values (Eqn. (18)), Alma is again poorest (Figs. 21 and 22), while Charleston shows the best agreement. However, relative differences among the sampling sites are small. Temperature indices are lower than wind indices, which is not surprising in view of the statistics already seen. A dip in the index of temperatures after sunrise is noted, also in agreement with Figs. 15 and 19. The Augusta location is clearly poorest after sunset for both sets of simulations (as discussed also with Fig. 17), while the other locations generally exhibit very similar indices of agreement over the entire forecast period. Temperature indices tend to be lower during the warmer months (Fig. 22), indicating larger variance in values relative to the observed mean, while indices for the winds show no apparent trends.

The figures discussed in this section indicate favorable model simulations. However, it should be noted that individual simulations could show very poor skill or realism. For example, Physick and Noonan (2000) show results for wind speed for a series of days over Hong Kong using the Penn State mesoscale model (MM5) and obtain values of $R_{R M S^{\sim}} 1.0, R_{S T D} \sim 0.7, I \sim 0.6$. In addition, Pielke and Mahrer (1978) obtain values of $R_{R M S} \sim 1.1$ and $R_{S T D} \sim 0.6$ to 0.95 for simulations performed in Florida. Willmott et al. (1985) calculate an agreement value $I \sim 0.8$ for springtime estimates of winds when compared with observations from a single buoy. The values in the current study appear to be better due to a much larger sampling time period and averaging over multiple forecast times or months. Nonetheless, these figures indicate that RAMS can skillfully predict the temperature and winds in terms of averaged quantities over entire months, or over entire forecast periods. Because the ratios of standard deviation of prediction to observation are near unity, the model also provides realistic values. In terms of simulating the meteorology, RAMS shows more skill and realism in predicting the winds compared with temperature. This is important due to the number of applications at SRS (emergency response, United States Forest Service control burns, and air quality) which rely on accurate wind speed and direction.

\section{CONCLUSIONS}

In summary, the prognostic model has difficulty with the transition from daytime to nocturnal conditions, particularly in the prediction of temperature and moisture fields. In general, it was seen that 00 GMT analyses were more skillful than the 12 GMT analyses. This is fortuitous for SRS operations, since most work is performed during the day, when results from the 00 GMT analyses would be utilized. 
There are numerous reasons for the model biases, as discussed in the introduction. In RAMS, temperature differences between the ground and the lowest atmospheric model levels affect surface fluxes of sensible heat, momentum and moisture, leading to changes in surface wind speeds. Overestimates in temperature result in higher heat and momentum fluxes, hence higher wind speeds, which is clearly seen in the results for Alma, Georgia (Figs. 9 and 11). Overall temperature errors seem to be highest during the cooler months for the inland locations (Augusta and Atlanta), indicating possible problems with surface budgets in the model. Deficiencies within the radiation and soil parameterizations, as well as the initial soil moisture selected for the model simulations all contribute to this error. There are numerous studies that address this very problem (e.g. Wilson et al. 1987, or Mahfouf et al. 1987). Of special concern is the large error that occurs during the transition from day to night (and vice-versa). Another issue deals with precipitation. Although RAMS can be configured to generate explicit precipitation, it is more time-consuming, which limits its use in an operational capacity. The operational version used at SRS generates precipitation through convective paramaterization, but does not incorporate explicit microphysics, leading to poorer results for those times in which rainfall occurs.

The large-scale models themselves contain biases. Manikin et al. (2000) discuss changes made to the convective scheme of the operational Eta model to reduce an over-abundance of forecasted precipitation over coastal regions of the Gulf and south Atlantic states, implemented in March, 2000. This may partly explain the frequent occurrence of poorly forecasted location of closed upper low-pressure systems approaching the Atlantic coast, which would lead to errors in numerous meteorological parameters. The large-scale model also has problems when the soil moisture it predicts is too high. This leads to overestimates in dew-point temperatures, and lowlevel temperatures that are too low, which often leads to an under-prediction in boundary layer winds. A cold bias in surface temperatures (due to insufficient near-surface vertical turbulent mixing) during calm, clear (stable) nights has been discussed as well, which is intensified especially during the winter months (Koren et al. 1999). This may in part explain the tendency for RAMS to underestimate cooler temperatures $\left(\leq 273^{\circ} \mathrm{K}\right.$, Figs. $\left.6 \mathrm{c}-\mathrm{e}\right)$.

Monthly averaged bias and RMS error information is available on the internet (http://lnx40.wwb.noaa.gov) for the Medium Range Forecast (MRF) model (of which the Aviation is a subset). Since inter-model biases (i.e. MRF, AVN, Eta) averaged over an entire month are not generally extremely variant, conclusions regarding the use of MRF model statistics are applied to RAMS results in the following discussion, even though RAMS is driven the majority of the time using the Eta large-scale model.

Examination of temperature biases of the MRF model when compared with upper-air soundings over all of North America for the period December 1998 to January 2000 indicate better agreement between February and June 1999 than for the other periods at the surface. This trend generally agrees with the RAMS simulated results shown in Fig. 11, especially for Augusta, Charleston, Alma, and Charlotte. In addition, monthly averaged biases for the MRF model using 00 GMT analyses ranged from $-0.2 \mathrm{~K}$ to $0.6 \mathrm{~K}$, whereas 12 GMT simulation analyses yielded biases between 0.7 and $1.1 \mathrm{~K}$ at the surface. Therefore, 00 GMT simulations of temperature 
provide better agreement. (This is also true for the wind speed biases). Since these large-scale models are driving RAMS, this explains the generally worse comparisons in standard deviation ratios, $R_{S T D}$, for the 12 GMT RAMS simulations (Fig. 19).

For upper-air results, wind speeds from the MRF at 500 mbar are consistently underestimated by 0.5 to $1.0 \mathrm{~m} \mathrm{~s}^{-1}$, which agrees with the RAMS underestimates of speed at this level (Fig. 13 and Table 6). On the other hand, the 300 mbar MRF bias for both 00 and 12 GMT analyses are consistently between 0.2 and $0.4 \mathrm{~K}$, which may provide a reason for the RAMS overestimates of temperature at both Atlanta and Charleston at this pressure level (Fig. 14 and Table 7). Thus, input conditions for mesoscale models play a crucial role in their forecast accuracy.

The RAMS simulations are valuable because they provide meteorological information in datasparse regions and as a forecast. The statistical comparisons discussed here have shown that the model can generate realistic results with skill. A major weakness is seen to be reliance on accurate input conditions (i.e. large-scale synoptic models) which drive the mesoscale model initial and lateral boundary conditions. One way to alleviate biases introduced as a result of large-scale model errors, is to use four-dimensional data assimilation (e.g. Stauffer and Seaman 1990). This will typically provide improved accuracy several hours beyond the initial simulation time period with the bulk of the measurements being restricted to the surface. The initial state of the atmosphere for the current RAMS simulations could be improved by using the local SRS tower data, which are available in 15-minute averages. This has already been done on an experimental basis (Fast et al. 1995) and would be particularly important for local transport applications.

An obvious improvement in RAMS simulations would be to refine the grid resolution. Recent upgrades to the computing facilities have resulted in much smaller real-time requirements for the simulations, allowing for the option of finer RAMS grids. In addition, large-scale analyses from NCEP continue to be refined, implying that the mesoscale model domain can be reduced as well. Implementation of a nested grid system to replace the current single 20-km horizontal grid spacing domain is underway. The new configuration contains a coarse grid spacing of $48 \mathrm{~km}$ spanning from the western edges of Tennessee, Kentucky and Alabama, eastward to the Atlantic Ocean, and from the northern edges of Kentucky and Virginia, southward to Miami, Florida. The inner grid has been reduced to 12-km horizontal spacing and covers the two-state region of Georgia and South Carolina, with forecasts generated out to 36 hours.

In addition to resolving more atmospheric features, use of a nested grid system will reduce "boundary effects" which occur along the outermost grid points of the coarse RAMS grid as a result of nudging RAMS meteorological fields to the large-scale forecasts interpolated in time (Warner et al, 1997). If RAMS results differ significantly from the large-scale forecasts, then contaminated solutions are possible. Therefore, the use of the nested-grid option has several advantages over the single-grid configuration. It should be cautioned, however, that increases in grid resolution do not necessarily imply better results. Hanna and Yang (2001) suggest that model-error reductions are limited due to random stochastic and turbulent fluctuations that can not be simulated. Although the results described in this report are encouraging, it is hoped that 
WSRC-TR-2001-00563

December 2001

future refinements to the mesoscale model in terms of grid structure, physics, and initialization will lead to an improvement in forecast capabilities. 


\section{REFERENCES}

Black T. L., 1994: The new NMC mesoscale Eta model: Description and forecast examples. Wea. Forecasting, 9, 265-278.

Cox R., B. L. Bauer, and T. Smith, 1998: A mesoscale model intercomparison. Bull. Amer. Met. Soc., 79, 265-283.

Davies, H. C., 1976: A lateral boundary formulation for multi-level prediction models. Quart. J. Roy. Met. Soc., 102, 405-418.

Dickinson R. E., A. Henderson-Sellers, P. J. Kennedy, and M. F. Wilson, 1986: BiosphereAtmosphere Transfer Scheme (BATS) for the NCAR Community Climate Model. National Center for Atmospheric Research, Tech. Note NCAR/TN-275+ST, 69 pp.

Dyer A. J. and B. B. Hicks, 1970: Flux-gradient relationships in the constant flux layer. Quart. J. Roy. Met. Soc., 96, 715-721.

Fast J. D., B. L. O'Steen, and R. P. Addis, 1995: Advanced atmospheric modeling for emergency response. J. Appl. Meteor., 34, 626-649.

Hanna S. R., and R. Yang, 2001: Evaluations of Mesoscale Models' Simulations of Near Surface Winds, Temperature Gradients, and Mixing Depths. J. Appl. Meteor., 40, 1095-1104.

Helfand H. M., and J. C. Labraga, 1988: Design of a nonsingular level 2.5 second-order closure model for the prediction of atmospheric turbulence. J. Atmos. Sci., 45, 113-132.

Keyser D. and R. A. Anthes, 1977: The applicability of a mixed-layer model of the planetary boundary layer to real-data forecasting. Mon. Wea. Rev., 105, 1351-1371.

Koren V., J. Schaake, K. Mitchell, Q-Y. Duan, F. Chen, and J. M. Baker, 1999: A parameterization of snowpack and frozen ground intended for NCEP weather and climate models. J. Geophys. Res., 104, (D16) 19569-19585.

Kurzeja R. J., 1993: The Savannah River Technology Center research and development climatology center. WSRC-TR-93-596, Westinghouse Savannah River Company, Aiken, SC.

Lascer A. and S. P. S. Arya, 1986: A comparative assessment of mixing-length parameterizations in the stably stratified nocturnal boundary layer (NBL). Bound.-Layer Meteor., 36, 53-70.

Lyons W. A., R. A. Pielke, C. J. Tremback, R. L. Walko, D. A. Moon, and C. S. Keen, 1995: Applications of the Regional Atmospheric Modeling System (RAMS) to provide input to photochemical grid models for the Lake Michigan Study (LMOS). J. Appl. Meteor., 34, 1762-1786. 
Mahfouf J.-F., E. Richard, and P. Mascart, 1987: The influence of soil and vegetation on the development of mesoscale circulations. J. Climate Appl. Meteor., 26, 1483-1495.

Manikin, G., M Baldwin, W. Collins, J. Gerrity, D. Keyser, Y. Lin, K. Mitchell, and E. Rogers, 2000 : Changes to the NCEP Meso Eta runs: Extended range, added input, added output, convective changes. NWS Technical Procedures Bulletin 465, NOAA/NWS. [Available at http://www.nws.noaa.gov/om/tpb/465.htm and from the National Weather Service, Office of Meteorology, 1325 East-West Highway. Silver Spring, MD 20910].

Manobianco J., J. W. Zack, and G. E. Taylor, 1996: Workstation-based real-time mesoscale modeling designed for weather support to operations at the Kennedy Space Center and Cape Canaveral Air Station. Bull. Amer. Meteor. Soc., 77 (4), 653-672.

McCumber M. C., and R. A. Pielke, 1981: Simulation of the effects of surface fluxes of heat and moisture in a mesoscale numerical model: I. Soil layer. J. Geophys. Res. 86 (C10), 99299938.

McQueen J. T., R. R. Draxler, and G. D. Rolph, 1995: Influence of grid size and terrain resolution on wind field predictions from an operational mesoscale model. J. Appl. Meteor., 34, 2166-2181.

Mellor G. L. and T. Yamada, 1982: Development of a turbulent closure model for geophysical fluid problems. Rev. Geophys. Space Phys. 20 (10), 851-875.

Mesinger F., Z. I. Janjic, S. Nickovic, D. Gavrilow, and D. G. Deaven, 1988: The step-mountain coordinate: model description and performance for cases of Alpine lee cyclogenesis and for a case of an Appalachian redevelopment. Mon. Wea. Rev., 116, 1493-1518.

Nappo, C., J. Y. Caneill, R. W. Furman, F. A. Gifford, J. C. Kaimal, M. L. Kramer, T. J. Lockhart, M. M. Pendergast, R. A. Pielke, D. Randerson, J. H. Shreffler, and J. C. Wyngaard, 1982: The Workshop on the Representativeness of Meteorological Observations, June 1981, Boulder, Colo. Bull. Amer. Meteor. Soc., 63, 761-764.

Panofsky, H. A. and J. A. Dutton, 1984: Atmospheric Turbulence: Models and Methods for Engineering Applications, John Wiley, New York, NY, pp. 119-155.

Pasquill F., 1983: Atmospheric Diffusion, John Wiley, New York, NY, pp. 222-232.

Physick W. L., and J. A. Noonan, 2000: Mesoscale modeling with MM5 for the PATH Study (Pollutants in the Atmosphere and their Transport over Hong Kong). $11^{\text {th }}$ Joint Conference on the Applications of Air Pollution Meteorology with the Air and Waste Management Association, $80^{\text {th }}$ Annual Meeting, American Meteorological Society, Long Beach, CA, 9095. 
Pielke R. A., W. R. Cotton, R. L. Walko, C. J. Tremback, W. A. Lyons, L. D. Grasso, M. E. Nicholls, M. D. Moran, D. A. Wesley, T. J. Lee, and J. H. Copeland, 1992: A comprehensive meteorological modeling system--RAMS. Meteor. Atmos. Phys., 49, 69-91.

Pielke, R. A. and Y. Mahrer, 1978: Verification analysis of the University of Virginia threedimensional mesoscale model prediction over South Florida for 1 July 1973. Mon. Wea. Rev., 106, 1568-1589.

Sela J., 1980: Spectral modeling at the National Meteorological Center. Mon. Wea. Rev., 108, 1279-1292.

Snook J. S., P. A. Stamus, J. Edwards, Z. Christidis, and J. A. McGinley, 1997: Local-domain mesoscale analysis and forecasting model support for the 1996 Summer Olympic Games. Wea. Forecasting, 13, 138-150.

Stauffer D. R. and N. L. Seaman, 1990: Use of four-dimensional data assimilation in a limited area mesoscale model. Part I: Experiments with synoptic-scale data. Mon. Wea. Rev., 118, 1250-1277.

Steyn D. G., and I. G. McKendry, 1988: Quantitative and qualitative evaluation of a threedimensional mesoscale numerical model simulation of a sea breeze in complex terrain. Mon. Wea. Rev., 116, 1914-1926.

Thom A. S.,1975: Momentum, mass and heat exchange of plant communities, in Vegetation and the Atmosphere, vol. 1., edited by J. L. Monteith, pp. 50-109, Academic, San Diego, CA.

Tremback C. J. and R. Kessler, 1985: A surface temperature and moisture parameterization for use in mesoscale numerical models. Preprints, Seventh Conf. on Numerical Weather Prediction, Montreal, Quebec, Canada, Amer. Meteor. Soc., 355-358.

Ulrickson B. L., and C. F. Mass, 1990: Numerical investigation of mesoscale circulations over the Los Angeles Basin. Part I. A verification study. Mon. Wea. Rev., 118, 2138-2161.

Warner T. T., R. A. Peterson, and R. E. Treadon, 1997: A tutorial on lateral boundary conditions as a basic and potentially serious limitation to regional numerical weather prediction. Bull. Amer. Meteor. Soc., 78, 2599-2617.

Weber A. H., J. S. Irwin, J. P. Kahler, and W. B. Petersen, 1975: Atmospheric turbulence properties in the lowest 300 meters. EPA-600/4-75-004, North Carolina State University, Raleigh, NC. 
Willmott C. J., S. G. Ackleson, R. E. Davis, J. J. Feddema, K. M. Klink, D. R. Legates, J. O'Donnell, and C. M. Rowe, 1985: Statistics for the evaluation and comparison of models. J. Geophys. Res., 90(C5), 8995-9005.

Wilson D. K, 2001: An alternative function for the wind and temperature gradients in unstable surface layers. Boundary-Layer Meteorol., 99, 151-158.

Wilson M. F., A. Henderson-Sellers, R. E. Dickinson, and P. J. Kennedy, 1987: Sensitivity of the biosphere-atmosphere transfer scheme (BATS) to the inclusion of variable soil characteristics. J. Climate Appl. Meteor., 26, 341-362. 
WSRC-TR-2001-00563

December 2001

Table 1: Sensitivity of Wind Speed Differences to Surface Roughness, $z_{0}$.

\begin{tabular}{|c|c|c|}
\hline $\boldsymbol{z}_{0}[\mathrm{~m}]$ & Mean Difference $\left[\mathbf{m ~ s}^{-1}\right]$ & Mean Absolute Difference $\left[\mathbf{m ~ s}^{\mathbf{- 1}}\right]$ \\
\hline 0.05 & 1.78 & 1.88 \\
\hline 0.10 & 1.26 & 1.49 \\
\hline 0.25 & 0.38 & 1.11 \\
\hline 0.50 & -0.50 & 1.03 \\
\hline
\end{tabular}


Table 2: Wind Direction Comparisons at the Surface

\begin{tabular}{|c|c|c|c|c|c|c|}
\hline CITY & $f[\mathbf{h r}]$ & $\mathbf{N}$ & $\begin{array}{c}\overline{|d|} \\
{[\operatorname{deg}]}\end{array}$ & $P_{15}[\%]$ & $P_{45}[\%]$ & $P_{75}[\%]$ \\
\hline \multirow[t]{5}{*}{ Atlanta } & 0 & 1029 & 32.4 & 39.2 & 76.8 & 89.8 \\
\hline & 6 & 965 & 33.9 & 33.7 & 75.1 & 89.9 \\
\hline & 12 & 1026 & 35.0 & 36.8 & 74.4 & 88.1 \\
\hline & 18 & 962 & 35.6 & 32.8 & 71.9 & 89.4 \\
\hline & 24 & 1023 & 36.8 & 34.6 & 72.6 & 86.0 \\
\hline \multirow[t]{5}{*}{ Augusta } & 0 & 723 & 35.2 & 34.3 & 74.4 & 86.9 \\
\hline & 6 & 781 & 36.6 & 32.1 & 72.2 & 88.1 \\
\hline & 12 & 722 & 36.6 & 34.3 & 72.7 & 86.1 \\
\hline & 18 & 784 & 40.6 & 31.5 & 68.2 & 82.9 \\
\hline & 24 & 722 & 38.1 & 32.5 & 70.8 & 85.3 \\
\hline \multirow[t]{5}{*}{ Alma } & 0 & 480 & 26.6 & 43.1 & 83.1 & 93.8 \\
\hline & 6 & 508 & 34.2 & 30.5 & 73.4 & 90.9 \\
\hline & 12 & 482 & 29.9 & 36.7 & 80.3 & 91.9 \\
\hline & 18 & 511 & 39.2 & 26.6 & 68.3 & 87.1 \\
\hline & 24 & 477 & 30.9 & 35.4 & 76.7 & 93.7 \\
\hline \multirow[t]{5}{*}{ Charleston } & 0 & 1125 & 29.9 & 41.3 & 80.4 & 91.4 \\
\hline & 6 & 1057 & 32.6 & 39.7 & 77.7 & 89.0 \\
\hline & 12 & 1133 & 33.1 & 37.2 & 77.4 & 89.2 \\
\hline & 18 & 1061 & 35.2 & 38.2 & 75.6 & 87.1 \\
\hline & 24 & 1133 & 35.5 & 36.5 & 74.1 & 87.3 \\
\hline \multirow[t]{5}{*}{ Charlotte } & 0 & 924 & 36.6 & 32.4 & 73.4 & 87.1 \\
\hline & 6 & 844 & 34.7 & 33.4 & 73.6 & 88.3 \\
\hline & 12 & 932 & 39.8 & 30.7 & 70.2 & 84.1 \\
\hline & 18 & 855 & 38.9 & 32.6 & 69.2 & 85.1 \\
\hline & 24 & 929 & 42.1 & 29.0 & 66.6 & 83.5 \\
\hline \multirow[t]{5}{*}{ SRS } & 0 & 801 & 31.2 & 39.3 & 78.4 & 91.0 \\
\hline & 6 & 800 & 33.1 & 36.8 & 75.5 & 89.5 \\
\hline & 12 & 801 & 36.2 & 36.2 & 73.7 & 86.4 \\
\hline & 18 & 800 & 37.4 & 32.4 & 71.9 & 86.3 \\
\hline & 24 & 801 & 38.3 & 33.5 & 70.3 & 84.9 \\
\hline
\end{tabular}

Note: $f=$ forecast hour, $N=$ Total number of points to compare, $\overline{|d|}=$ mean absolute difference [deg], $P_{15}=$ percentage of points with absolute error $\leq 15^{\circ}, P_{45}=$ percentage of points with absolute error $\leq 45^{\circ}, P_{75}=$ percentage of points with absolute error $\leq 75^{\circ}$. 
Table 3: Wind Speed Comparisons at the Surface

\begin{tabular}{|c|c|c|c|c|c|c|c|c|}
\hline CITY & $\underset{[\mathbf{h r}]}{f}$ & $\mathbf{N}$ & $\begin{array}{c}\bar{d} \\
{\left[\mathrm{~m} \mathrm{~s}^{-1}\right]}\end{array}$ & $C I_{M, 95}$ & $\begin{array}{c}\sigma_{\text {Diff }} \\
{\left[\mathrm{m} \mathrm{s}^{-1}\right]}\end{array}$ & $C I_{S, 95}$ & $\begin{array}{c}\overline{|d|} \\
{\left[\mathrm{m} \mathrm{s}^{-1}\right]}\end{array}$ & $\begin{array}{c}P_{1} \\
{[\%]}\end{array}$ \\
\hline \multirow[t]{5}{*}{ ATL } & 0 & 1029 & -0.583 & {$[-.670,-.496]$} & 1.43 & {$[1.37,1.49]$} & 1.17 & 59.8 \\
\hline & 6 & 965 & -0.359 & {$[-.458,-.259]$} & 1.57 & {$[1.51,1.65]$} & 1.23 & 63.6 \\
\hline & 12 & 1026 & -0.478 & {$[-.570,-.387]$} & 1.50 & {$[1.43,1.56]$} & 1.20 & 60.2 \\
\hline & 18 & 962 & -0.323 & {$[-.426,-.219]$} & 1.63 & {$[1.56,1.71]$} & 1.28 & 61.9 \\
\hline & 24 & 1023 & -0.452 & {$[-.547,-.357]$} & 1.55 & {$[1.49,1.62]$} & 1.23 & 62.1 \\
\hline \multirow[t]{5}{*}{ AUG } & 0 & 723 & 0.271 & {$[.178, .364]$} & 1.27 & {$[1.21,1.34]$} & 1.00 & 80.5 \\
\hline & 6 & 781 & 0.173 & {$[.0620, .284]$} & 1.58 & {$[1.51,1.66]$} & 1.23 & 67.7 \\
\hline & 12 & 722 & 0.331 & {$[.232, .431]$} & 1.36 & {$[1.29,1.43]$} & 1.08 & 76.5 \\
\hline & 18 & 784 & 0.092 & [.0244, .209] & 1.66 & {$[1.58,1.75]$} & 1.30 & 66.5 \\
\hline & 24 & 722 & 0.365 & {$[.261, .470]$} & 1.43 & {$[1.36,1.50]$} & 1.13 & 74.5 \\
\hline \multirow[t]{5}{*}{ ALM } & 0 & 482 & 0.780 & {$[.655, .904]$} & 1.40 & {$[1.31,1.49]$} & 1.29 & 75.1 \\
\hline & 6 & 510 & 0.938 & {$[.788,1.09]$} & 1.72 & {$[1.62,1.84]$} & 1.56 & 63.5 \\
\hline & 12 & 482 & 0.871 & {$[.739,1.00]$} & 1.48 & {$[1.39,1.58]$} & 1.37 & 73.0 \\
\hline & 18 & 513 & 0.906 & {$[.748,1.06]$} & 1.81 & {$[1.71,1.93]$} & 1.63 & 59.1 \\
\hline & 24 & 479 & 0.862 & {$[.732, .992]$} & 1.45 & {$[1.36,1.55]$} & 1.36 & 70.8 \\
\hline \multirow[t]{5}{*}{$\mathrm{CHS}$} & 0 & 1125 & 0.039 & {$[-.0360, .113]$} & 1.27 & {$[1.22,1.33]$} & 0.98 & 76.6 \\
\hline & 6 & 1057 & 0.128 & {$[.0390, .218]$} & 1.48 & {$[1.42,1.55]$} & 1.12 & 72.0 \\
\hline & 12 & 1133 & 0.141 & {$[.0609, .221]$} & 1.38 & {$[1.32,1.44]$} & 1.07 & 74.0 \\
\hline & 18 & 1061 & 0.075 & {$[-.0192, .169]$} & 1.56 & {$[1.50,1.63]$} & 1.18 & 70.6 \\
\hline & 24 & 1133 & 0.132 & {$[.0446, .220]$} & 1.51 & {$[1.45,1.57]$} & 1.15 & 71.4 \\
\hline \multirow[t]{5}{*}{ CHR } & 0 & 924 & -0.055 & {$[-.131, .0221]$} & 1.19 & {$[1.13,1.24]$} & 0.90 & 77.2 \\
\hline & 6 & 844 & 0.106 & {$[.0109, .0202]$} & 1.41 & {$[1.35,1.48]$} & 1.09 & 70.3 \\
\hline & 12 & 932 & -0.026 & {$[-.110, .0591]$} & 1.32 & {$[1.26,1.38]$} & 1.00 & 74.2 \\
\hline & 18 & 855 & 0.157 & {$[.0556, .0259]$} & 1.51 & {$[1.44,1.59]$} & 1.16 & 69.4 \\
\hline & 24 & 929 & -0.026 & {$[-.111, .0597]$} & 1.33 & {$[1.27,1.39]$} & 1.00 & 74.0 \\
\hline \multirow[t]{5}{*}{ SRS } & 0 & 801 & 0.376 & {$[.302, .449]$} & 1.07 & {$[1.02,1.12]$} & 0.86 & 84.0 \\
\hline & 6 & 800 & 0.658 & {$[.562, .754]$} & 1.39 & {$[1.32,1.46]$} & 1.17 & 75.0 \\
\hline & 12 & 801 & 0.488 & {$[.405, .570]$} & 1.19 & {$[1.13,1.25]$} & 0.99 & 82.1 \\
\hline & 18 & 800 & 0.635 & {$[.537, .734]$} & 1.42 & {$[1.36,1.50]$} & 1.20 & 73.6 \\
\hline & 24 & 801 & 0.479 & {$[.393, .566]$} & 1.25 & {$[1.19,1.31]$} & 1.02 & 80.4 \\
\hline
\end{tabular}

Note: $f=$ forecast hour, $N=$ Total number of points to compare, $\bar{d}=$ mean difference between simulation and observation, $C I_{M, 95}=95 \%$ confidence interval for $\bar{d}, \sigma_{D i f f}=$ standard deviation of the difference, $C I_{S, 95}=95 \%$ confidence interval for $\sigma_{D i f f}, \overline{|d|}=$ mean absolute difference, $P_{1}=$ percentage of points falling within a range of $\pm 1 \mathrm{~m} \mathrm{~s}^{-1}$. 
Table 4: Temperature Comparisons at the Surface

\begin{tabular}{|c|c|c|c|c|c|c|c|c|}
\hline CITY & $\underset{[\mathbf{h r}]}{f}$ & $\mathbf{N}$ & $\begin{array}{c}\overline{\boldsymbol{d}} \\
{[\mathbf{K}]}\end{array}$ & $C I_{M, 95}$ & $\begin{array}{l}\sigma_{\text {Diff }} \\
{[\mathbf{K}]}\end{array}$ & $C I_{S, 95}$ & $\begin{array}{l}\overline{|d|} \\
{[\mathrm{K}]}\end{array}$ & $\begin{array}{c}P_{2} \\
{[\%]}\end{array}$ \\
\hline \multirow[t]{5}{*}{ ATL } & 0 & 1283 & 0.504 & {$[.378, .631]$} & 2.31 & {$[2.23,2.40]$} & 1.77 & 75.4 \\
\hline & 6 & 1282 & -0.064 & {$[-.208, .0810]$} & 2.64 & {$[2.54,2.75]$} & 2.03 & 68.1 \\
\hline & 12 & 1290 & 0.181 & {$[.0295, .333]$} & 2.78 & {$[2.68,2.89]$} & 2.12 & 66.7 \\
\hline & 18 & 1287 & -0.256 & {$[-.430,-.0820]$} & 3.18 & {$[3.06,3.31]$} & 2.41 & 62.1 \\
\hline & 24 & 1289 & 0.076 & {$[-.0879, .241]$} & 3.01 & {$[2.89,3.13]$} & 2.27 & 64.3 \\
\hline \multirow[t]{5}{*}{ AUG } & 0 & 1263 & 2.021 & {$[1.87,2.17]$} & 2.75 & {$[2.65,2.87]$} & 2.65 & 61.0 \\
\hline & 6 & 1263 & 0.509 & {$[.310, .708]$} & 3.60 & {$[3.47,3.75]$} & 2.94 & 48.9 \\
\hline & 12 & 1271 & 1.833 & {$[1.68,1.99]$} & 2.82 & {$[2.71,2.93]$} & 2.67 & 58.7 \\
\hline & 18 & 1268 & 0.073 & {$[-.159, .304]$} & 4.20 & {$[4.04,4.37]$} & 3.25 & 48.1 \\
\hline & 24 & 1271 & 1.624 & {$[1.46,1.79]$} & 2.97 & {$[2.85,3.09]$} & 2.68 & 58.3 \\
\hline \multirow[t]{5}{*}{ ALM } & 0 & 1207 & 1.924 & {$[1.78,2.07]$} & 2.55 & {$[2.45,2.66]$} & 2.48 & 65.2 \\
\hline & 6 & 614 & -0.713 & {$[-.931,-.496]$} & 2.74 & {$[2.60,2.91]$} & 2.16 & 60.3 \\
\hline & 12 & 1216 & 1.752 & {$[1.62,1.89]$} & 2.44 & {$[2.35,2.54]$} & 2.38 & 66.2 \\
\hline & 18 & 612 & -1.248 & {$[-1.55,-.948]$} & 3.77 & {$[3.57,3.99]$} & 2.86 & 54.4 \\
\hline & 24 & 1214 & 1.654 & {$[1.51,1.80]$} & 2.59 & {$[2.49,2.70]$} & 2.38 & 66.1 \\
\hline \multirow[t]{5}{*}{$\mathrm{CHS}$} & 0 & 1280 & 1.594 & {$[1.47,1.72]$} & 2.27 & {$[2.18,2.36]$} & 2.09 & 74.1 \\
\hline & 6 & 1277 & 0.350 & {$[.195, .505]$} & 2.83 & {$[2.72,2.94]$} & 2.23 & 63.6 \\
\hline & 12 & 1288 & 1.274 & {$[1.15,1.40]$} & 2.34 & {$[2.25,2.43]$} & 2.04 & 71.7 \\
\hline & 18 & 1284 & 0.138 & {$[-.0397, .315]$} & 3.24 & {$[3.12,3.37]$} & 2.51 & 60.1 \\
\hline & 24 & 1288 & 1.156 & {$[1.02,1.30]$} & 2.54 & {$[2.44,2.64]$} & 2.12 & 70.2 \\
\hline \multirow[t]{5}{*}{ CHR } & 0 & 1281 & 0.811 & {$[.667, .955]$} & 2.63 & {$[2.53,2.73]$} & 2.11 & 65.7 \\
\hline & 6 & 1272 & -0.213 & {$[-.371,-.0559]$} & 2.86 & {$[2.76,2.98]$} & 2.26 & 61.5 \\
\hline & 12 & 1287 & 0.455 & {$[.293, .616]$} & 2.95 & {$[2.84,3.07]$} & 2.30 & 61.7 \\
\hline & 18 & 1278 & -0.475 & {$[-.660,-.290]$} & 3.37 & {$[3.25,3.51]$} & 2.54 & 61.3 \\
\hline & 24 & 1287 & 0.257 & {$[.0767, .436]$} & 3.29 & {$[3.17,3.42]$} & 2.55 & 57.9 \\
\hline \multirow[t]{5}{*}{ SRS } & 0 & 804 & 0.675 & {$[.501, .850]$} & 2.52 & {$[2.40,2.65]$} & 1.99 & 72.4 \\
\hline & 6 & 802 & 0.539 & {$[.358, .719]$} & 2.61 & {$[2.49,2.74]$} & 2.02 & 71.3 \\
\hline & 12 & 804 & 0.411 & {$[.210, .612]$} & 2.90 & {$[2.77,3.05]$} & 2.26 & 66.9 \\
\hline & 18 & 802 & 0.009 & {$[-.221, .239]$} & 3.32 & {$[3.17,3.49]$} & 2.42 & 64.0 \\
\hline & 24 & 804 & 0.182 & {$[-.0359, .400]$} & 3.15 & {$[3.00,3.31]$} & 2.42 & 62.3 \\
\hline
\end{tabular}

Note: $f=$ forecast hour, $N=$ Total number of points to compare, $\bar{d}=$ mean difference between simulation and observation, $C I_{M, 95}=95 \%$ confidence interval for $\bar{d}, \sigma_{D i f f}=$ standard deviation of the difference, $C I_{S, 95}=95 \%$ confidence interval for $\sigma_{D i f f}, \overline{|d|}=$ mean absolute difference, $P_{2}=$ percentage of points falling within a range of \pm 2 degrees. 
Table 5: Wind Direction Comparisons at Upper-Air Levels

\begin{tabular}{|c|c|c|c|c|c|c|}
\hline CITY & $f[\mathbf{h r}]$ & $\mathbf{N}$ & $\overline{|d|}$ [deg] & $P_{15}[\%]$ & $P_{45}[\%]$ & $P_{75}[\%]$ \\
\hline ATL & 0 & 1170 & 23.7 & 50.6 & 87.2 & 93.6 \\
\hline \multirow{2}{*}{$(850 \mathrm{mb})$} & 12 & 1175 & 25.2 & 49.5 & 85.5 & 93.6 \\
\hline & 24 & 1018 & 28.2 & 44.3 & 82.0 & 91.9 \\
\hline $\mathrm{CHS}$ & 0 & 1167 & 26.3 & 46.4 & 83.8 & 92.6 \\
\hline \multirow[t]{2}{*}{$(850 \mathrm{mb})$} & 12 & 1172 & 27.9 & 45.8 & 81.8 & 91.5 \\
\hline & 24 & 993 & 29.3 & 44.4 & 80.0 & 90.7 \\
\hline ATL & 0 & 1189 & 19.9 & 61.5 & 88.9 & 95.2 \\
\hline \multirow[t]{2}{*}{$(700 \mathrm{mb})$} & 12 & 1194 & 22.1 & 57.5 & 87.4 & 94.2 \\
\hline & 24 & 1032 & 24.0 & 53.7 & 86.0 & 93.4 \\
\hline CHS & 0 & 1161 & 21.0 & 58.7 & 89.2 & 95.0 \\
\hline \multirow[t]{2}{*}{$(700 \mathrm{mb})$} & 12 & 1167 & 22.0 & 55.1 & 88.0 & 94.6 \\
\hline & 24 & 995 & 24.3 & 51.4 & 86.9 & 93.2 \\
\hline ATL & 0 & 1179 & 13.4 & 75.7 & 94.1 & 97.7 \\
\hline \multirow[t]{2}{*}{$(500 \mathrm{mb})$} & 12 & 1188 & 16.0 & 70.1 & 92.9 & 96.8 \\
\hline & 24 & 1024 & 17.8 & 65.2 & 91.5 & 96.1 \\
\hline CHS & 0 & 1144 & 14.6 & 70.9 & 94.4 & 97.1 \\
\hline \multirow[t]{2}{*}{$(500 \mathrm{mb})$} & 12 & 1149 & 16.5 & 67.4 & 92.6 & 96.4 \\
\hline & 24 & 977 & 18.4 & 62.8 & 91.4 & 96.5 \\
\hline ATL & 0 & 1163 & 11.0 & 81.2 & 96.9 & 98.7 \\
\hline \multirow[t]{2}{*}{$(300 \mathrm{mb})$} & 12 & 1168 & 12.6 & 75.4 & 95.3 & 98.5 \\
\hline & 24 & 1013 & 15.2 & 70.0 & 93.8 & 97.1 \\
\hline CHS & 0 & 1097 & 12.5 & 78.1 & 95.5 & 98.2 \\
\hline \multirow[t]{2}{*}{$(300 \mathrm{mb})$} & 12 & 1100 & 14.0 & 73.0 & 94.5 & 98.1 \\
\hline & 24 & 941 & 15.1 & 69.3 & 94.3 & 97.4 \\
\hline
\end{tabular}

Note: $f=$ forecast hour, $N=$ Total number of points to compare, $\overline{|d|}=$ mean absolute difference [deg], $P_{15}=$ percentage of points with absolute error $\leq 15^{\circ}, P_{45}=$ percentage of points with absolute error $\leq 45^{\circ}, P_{75}=$ percentage of points with absolute error $\leq 75^{\circ}$. 
Table 6: Wind Speed Comparisons at Upper-Air Levels

\begin{tabular}{|c|c|c|c|c|c|c|c|c|}
\hline CITY & $\begin{array}{c}f \\
{[\mathbf{h r}]}\end{array}$ & $\mathbf{N}$ & $\begin{array}{c}\bar{d} \\
{\left[\mathrm{~m} \mathrm{~s}^{-1}\right]}\end{array}$ & $C I_{M, 95}$ & $\begin{array}{c}\sigma_{D i f f} \\
{\left[\mathrm{~m} \mathrm{~s}^{-1}\right]}\end{array}$ & $C I_{S, 95}$ & $\begin{array}{c}\overline{|d|} \\
{\left[\mathrm{m} \mathrm{s}^{-1}\right]}\end{array}$ & $\begin{array}{l}P_{2.5} \\
{[\%]}\end{array}$ \\
\hline ATL & 0 & 1170 & -0.373 & {$[-.524,-.222]$} & 2.64 & {$[2.53,2.75]$} & 1.98 & 80.4 \\
\hline \multirow[t]{2}{*}{$(850 \mathrm{mb})$} & 12 & 1175 & -0.0417 & {$[-.210, .127]$} & 2.94 & {$[2.83,3.07]$} & 2.25 & 77.6 \\
\hline & 24 & 1018 & 0.0713 & {$[-.130, .273]$} & 3.27 & {$[3.14,3.42]$} & 2.52 & 74.5 \\
\hline $\mathrm{CHS}$ & 0 & 1167 & -0.431 & {$[-.590,-.271]$} & 2.77 & {$[2.66,2.89]$} & 2.14 & 77.6 \\
\hline \multirow[t]{2}{*}{$(850 \mathrm{mb})$} & 12 & 1172 & -0.199 & {$[-.381,-.0172]$} & 3.17 & {$[3.05,3.31]$} & 2.35 & 76.6 \\
\hline & 24 & 993 & -0.0571 & {$[-.271, .157]$} & 3.43 & {$[3.29,3.59]$} & 2.58 & 72.6 \\
\hline ATL & 0 & 1189 & -0.509 & {$[-.666,-.353]$} & 2.76 & {$[2.65,2.87]$} & 2.05 & 79.0 \\
\hline \multirow[t]{2}{*}{$(700 \mathrm{mb})$} & 12 & 1194 & -0.335 & {$[-.511,-.159]$} & 3.10 & {$[2.98,3.23]$} & 2.35 & 76.1 \\
\hline & 24 & 1032 & -0.322 & {$[-.528,-.115]$} & 3.39 & {$[3.25,3.54]$} & 2.61 & 72.7 \\
\hline CHS & 0 & 1161 & -0.620 & {$[-.777,-.462]$} & 2.74 & {$[2.63,2.86]$} & 2.13 & 76.7 \\
\hline \multirow{2}{*}{$(700 \mathrm{mb})$} & 12 & 1167 & -0.733 & {$[-.915,-.550]$} & 3.18 & {$[3.05,3.31]$} & 2.45 & 72.2 \\
\hline & 24 & 995 & -0.592 & {$[-.814,-.371]$} & 3.56 & {$[3.41,3.72]$} & 2.72 & 68.1 \\
\hline ATL & 0 & 1179 & -0.477 & {$[-.648,-.306]$} & 2.99 & {$[2.87,3.12]$} & 2.22 & 75.6 \\
\hline \multirow[t]{2}{*}{$(500 \mathrm{mb})$} & 12 & 1188 & -0.849 & {$[-1.04,-.654]$} & 3.42 & {$[3.29,3.57]$} & 2.65 & 68.9 \\
\hline & 24 & 1024 & -0.894 & {$[-1.13,-.659]$} & 3.83 & {$[3.67,4.00]$} & 3.01 & 62.3 \\
\hline $\mathrm{CHS}$ & 0 & 1144 & -0.544 & {$[-.728,-.359]$} & 3.18 & {$[3.05,3.31]$} & 2.30 & 76.4 \\
\hline \multirow[t]{2}{*}{$(500 \mathrm{mb})$} & 12 & 1149 & -0.993 & {$[-1.20,-.788]$} & 3.54 & {$[3.40,3.69]$} & 2.63 & 68.8 \\
\hline & 24 & 977 & -1.10 & {$[-1.34,-.849]$} & 3.93 & {$[3.76,4.11]$} & 2.88 & 66.7 \\
\hline ATL & 0 & 1163 & 0.178 & {$[-.100, .457]$} & 4.84 & {$[4.65,5.04]$} & 3.28 & 67.4 \\
\hline \multirow[t]{2}{*}{$(300 \mathrm{mb})$} & 12 & 1168 & 0.0524 & {$[-.258, .362]$} & 5.40 & {$[5.19,5.63]$} & 3.86 & 59.1 \\
\hline & 24 & 1013 & 0.0914 & {$[-.285, .468]$} & 6.10 & {$[5.85,6.38]$} & 4.46 & 52.8 \\
\hline CHS & 0 & 1097 & -0.0412 & {$[-.287, .204]$} & 4.14 & {$[3.98,4.32]$} & 3.05 & 67.3 \\
\hline \multirow[t]{2}{*}{$(300 \mathrm{mb})$} & 12 & 1100 & -0.464 & {$[-.744,-.183]$} & 4.74 & {$[4.55,4.95]$} & 3.53 & 59.5 \\
\hline & 24 & 941 & -0.628 & {$[-.971,-.286]$} & 5.35 & {$[5.12,5.60]$} & 4.04 & 54.0 \\
\hline
\end{tabular}

Note: $f=$ forecast hour, $N=$ Total number of points to compare, $\bar{d}=$ mean difference between simulation and observation, $C I_{M, 95}=95 \%$ confidence interval for $\bar{d}, \sigma_{\text {Diff }}=$ standard deviation of the difference, $C I_{S, 95}=95 \%$ confidence interval for $\sigma_{D i f f}, \overline{|d|}=$ mean absolute difference, $P_{2.5}=$ percentage of points falling within a range of $\pm 2.5 \mathrm{~m} \mathrm{~s}^{-1}$. 
Table 7: Temperature Comparisons at Upper-Air Levels

\begin{tabular}{|c|c|c|c|c|c|c|c|c|}
\hline CITY & $\underset{[\mathbf{h r}]}{f}$ & $\mathbf{N}$ & $\begin{array}{c}\overline{\boldsymbol{d}} \\
{[\mathbf{K}]}\end{array}$ & $C I_{M, 95}$ & $\begin{array}{l}\sigma_{\text {Diff }} \\
{[\mathbf{K}]}\end{array}$ & $C I_{S, 95}$ & $\begin{array}{l}\overline{|\boldsymbol{d}|} \\
{[\mathbf{K}]}\end{array}$ & $\begin{array}{c}\boldsymbol{P}_{2} \\
{[\%]}\end{array}$ \\
\hline ATL & 0 & 1219 & 0.0906 & {$[.0093, .172]$} & 1.45 & {$[1.39,1.51]$} & 1.00 & 93.5 \\
\hline \multirow[t]{2}{*}{$(850 \mathrm{mb})$} & 12 & 1223 & 0.103 & {$[.0136, .192]$} & 1.59 & {$[1.53,1.66]$} & 1.13 & 89.0 \\
\hline & 24 & 1063 & 0.138 & {$[.0312, .245]$} & 1.77 & {$[1.70,1.85]$} & 1.29 & 86.7 \\
\hline $\mathrm{CHS}$ & 0 & 1218 & -0.0818 & {$[-.151,-.0066]$} & 1.34 & {$[1.29,1.39]$} & 0.957 & 94.1 \\
\hline \multirow[t]{2}{*}{$(850 \mathrm{mb})$} & 12 & 1225 & -0.239 & {$[-.323,-.154]$} & 1.51 & {$[1.45,1.57]$} & 1.11 & 90.0 \\
\hline & 24 & 1046 & -0.241 & {$[-.340,-.142]$} & 1.63 & {$[1.56,1.70]$} & 1.20 & 88.2 \\
\hline ATL & 0 & 1217 & -0.222 & {$[-.283,-.162]$} & 1.08 & {$[1.03,1.12]$} & 0.842 & 94.6 \\
\hline \multirow[t]{2}{*}{$(700 \mathrm{mb})$} & 12 & 1222 & -0.492 & {$[-.562,-.423]$} & 1.24 & {$[1.19,1.29]$} & 1.03 & 89.5 \\
\hline & 24 & 1062 & -0.683 & {$[-.769,-.598]$} & 1.42 & {$[1.36,1.48]$} & 1.21 & 83.1 \\
\hline CHS & 0 & 1215 & -0.256 & {$[-.322,-.190]$} & 1.17 & {$[1.13,1.23]$} & 0.882 & 93.8 \\
\hline \multirow{2}{*}{$(700 \mathrm{mb})$} & 12 & 1222 & -0.515 & {$[-.590,-.440]$} & 1.34 & {$[1.29,1.40]$} & 1.07 & 88.8 \\
\hline & 24 & 1044 & -0.750 & {$[-.836,-.665]$} & 1.41 & {$[1.35,1.47]$} & 1.22 & 83.6 \\
\hline ATL & 0 & 1211 & 0.123 & {$[.0703, .176]$} & 0.935 & {$[.899, .974]$} & 0.706 & 98.3 \\
\hline \multirow[t]{2}{*}{$(500 \mathrm{mb})$} & 12 & 1216 & 0.0128 & {$[-.0464, .072]$} & 1.05 & {$[1.01,1.10]$} & 0.796 & 97.4 \\
\hline & 24 & 1056 & -0.0494 & {$[-.119, .0198]$} & 1.15 & {$[1.10,1.20]$} & 0.858 & 95.1 \\
\hline CHS & 0 & 1206 & 0.0459 & {$[-.0218, .114]$} & 1.20 & {$[1.15,1.25]$} & 0.702 & 97.9 \\
\hline \multirow[t]{2}{*}{$(500 \mathrm{mb})$} & 12 & 1213 & -0.0890 & {$[-.162,-.0157]$} & 1.30 & {$[1.25,1.36]$} & 0.807 & 96.5 \\
\hline & 24 & 1037 & -0.105 & {$[-.191,-.0187]$} & 1.41 & {$[1.35,1.48]$} & 0.904 & 95.4 \\
\hline ATL & 0 & 1196 & 0.669 & {$[.613, .725]$} & 0.986 & {$[.948,1.03]$} & 0.928 & 97.7 \\
\hline \multirow[t]{2}{*}{$(300 \mathrm{mb})$} & 12 & 1200 & 0.626 & {$[.560, .692]$} & 1.16 & {$[1.12,1.21]$} & 1.02 & 95.9 \\
\hline & 24 & 1045 & 0.623 & {$[.544, .703]$} & 1.30 & {$[1.25,1.37]$} & 1.09 & 93.8 \\
\hline CHS & 0 & 1170 & 0.379 & {$[.317, .441]$} & 1.08 & {$[1.04,1.12]$} & 0.816 & 97.5 \\
\hline \multirow[t]{2}{*}{$(300 \mathrm{mb})$} & 12 & 1175 & 0.307 & {$[.235, .379]$} & 1.26 & {$[1.21,1.32]$} & 0.914 & 96.2 \\
\hline & 24 & 1009 & 0.338 & {$[.258, .418]$} & 1.30 & {$[1.24,1.36]$} & 1.01 & 95.3 \\
\hline
\end{tabular}

Note: $f=$ forecast hour, $N=$ Total number of points to compare, $\bar{d}=$ mean difference between simulation and observation, $C I_{M, 95}=95 \%$ confidence interval for $\bar{d}, \sigma_{\text {Diff }}=$ standard deviation of the difference, $C I_{S, 95}=95 \%$ confidence interval for $\sigma_{D i f f}, \overline{|d|}=$ mean absolute difference, $P_{2}=$ percentage of points falling within a range of \pm 2 degrees. 
WSRC-TR-2001-00563

December 2001

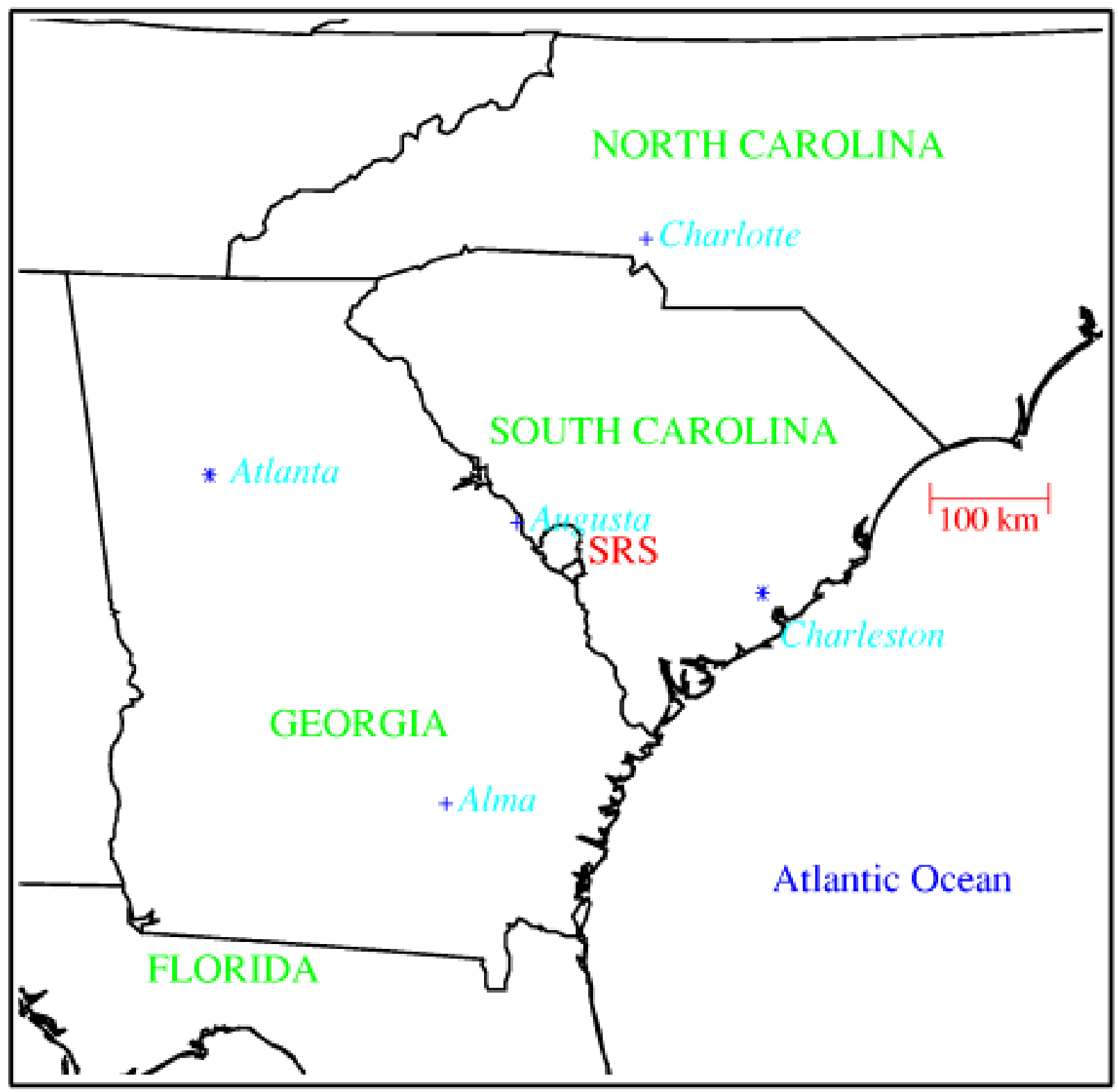

Figure 1: RAMS domain used in the applications at the SRS. The five cities from which statistical comparisons are made (along with the SRS) are indicated. Upper-air comparisons are also made at Atlanta and Charleston. 


\section{Simulation Period: April 1998 - March 2000}
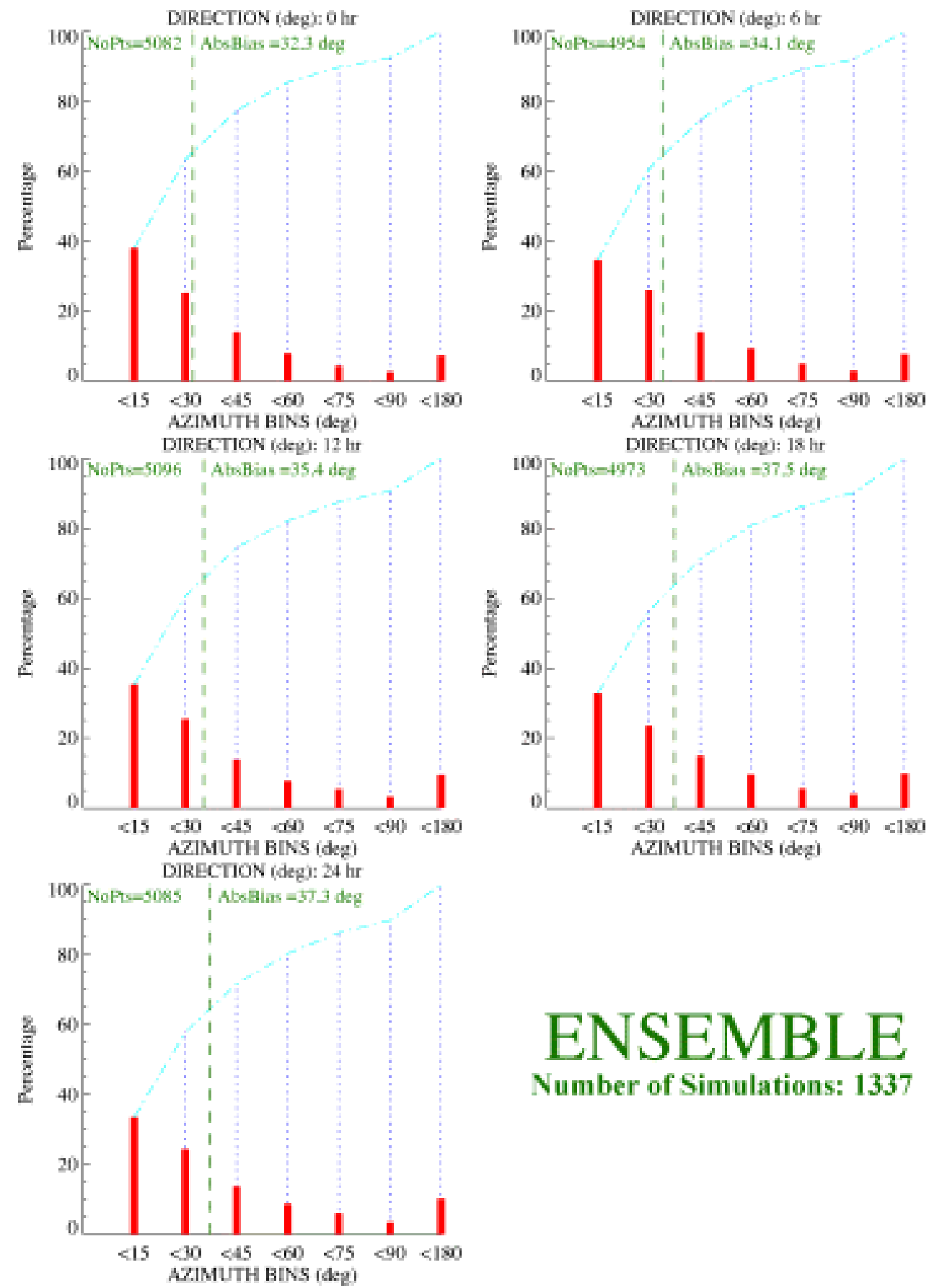

\section{ENSEMBLE}

Number of Simulations: 1337

Figure 2: Histogram plots showing wind direction (deg) comparisons for all six locations at the surface for a forecast period of: (a) 0-hr, (b) 6-hr, (c) 12-hr, (d) 18-hr, (e) 24-hr. For each forecast period, an absolute difference constrained to be between 0 and $180^{\circ}$ is calculated and the percentage of bin values in degrees is shown. The cumulative amount for a given error range is given by the dash-dot line connecting the various bin levels. In addition, "No.Pts." is the number of times represented in the plot, while "AbsBias" indicates the mean absolute difference for that particular forecast time. 


\section{Simulation Period: April 1998 - March 2000}
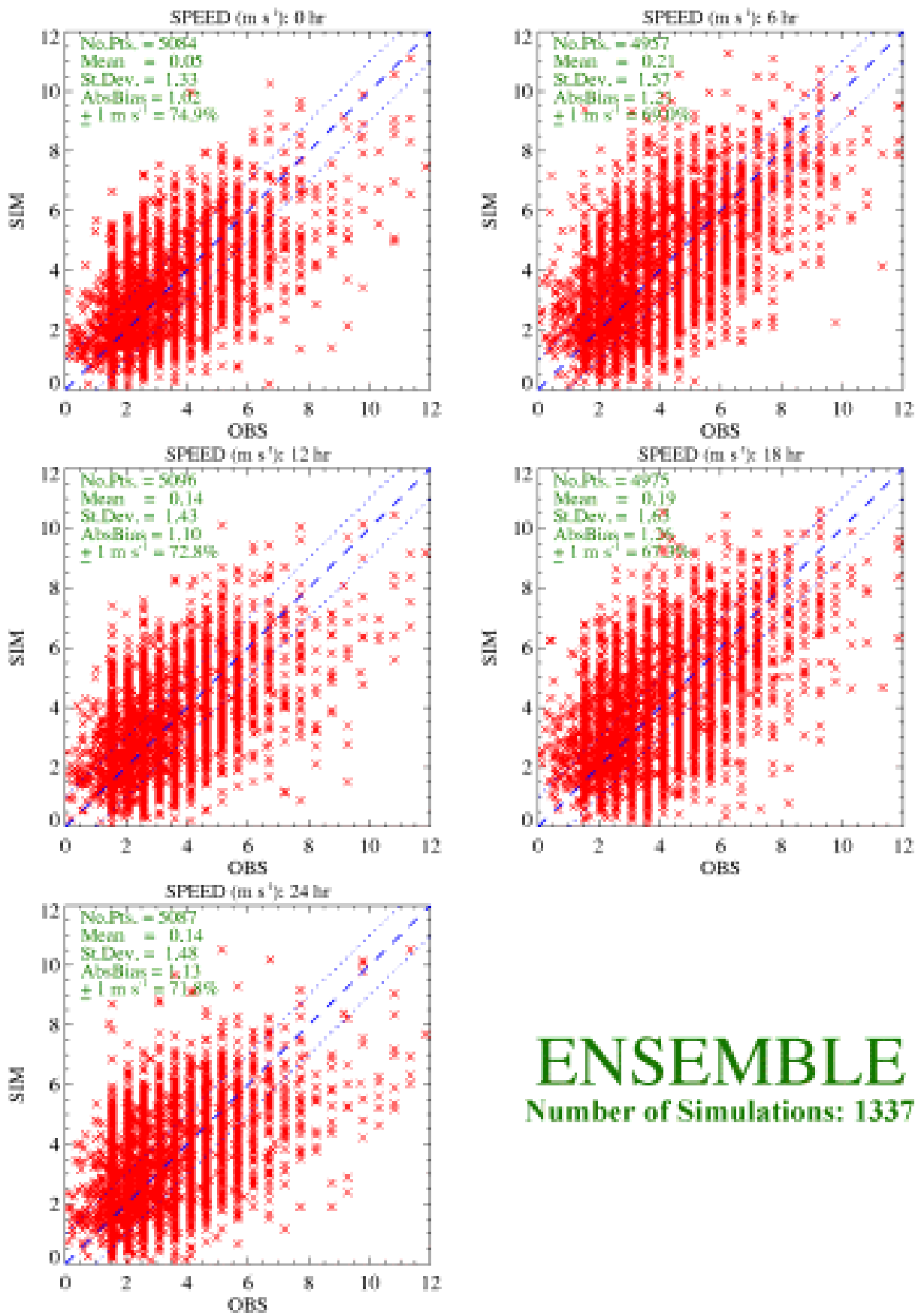

Number of Simulations: 1337

Figure 3: Scatter plot showing surface wind speed $\left(\mathrm{m} \mathrm{s}^{-1}\right)$ comparisons for the six locations for a forecast period of: (a) 0-hr, (b) 6-hr, (c) 12-hr, (d) 18-hr, (e) 24-hr. The observed quantity is shown along the $x$-axis, and the simulated quantity is shown along the $y$-axis. The bracketing value is $1 \mathrm{~m} \mathrm{~s}^{-1}$ to either side of the $45^{\circ}$ line. For each forecast period, the following are given: "No.Pts.", the number of times represented in the plot, "Mean", the mean difference between simulated and observed wind speed, "St.Dev.", the standard deviation, "AbsBias", the mean absolute bias, and the percentage of times in which the simulated wind speed was within $\pm 1 \mathrm{~m} \mathrm{~s}^{-1}$ of the observed value. 


\section{Simulation Period: February 1999 - March 2000}
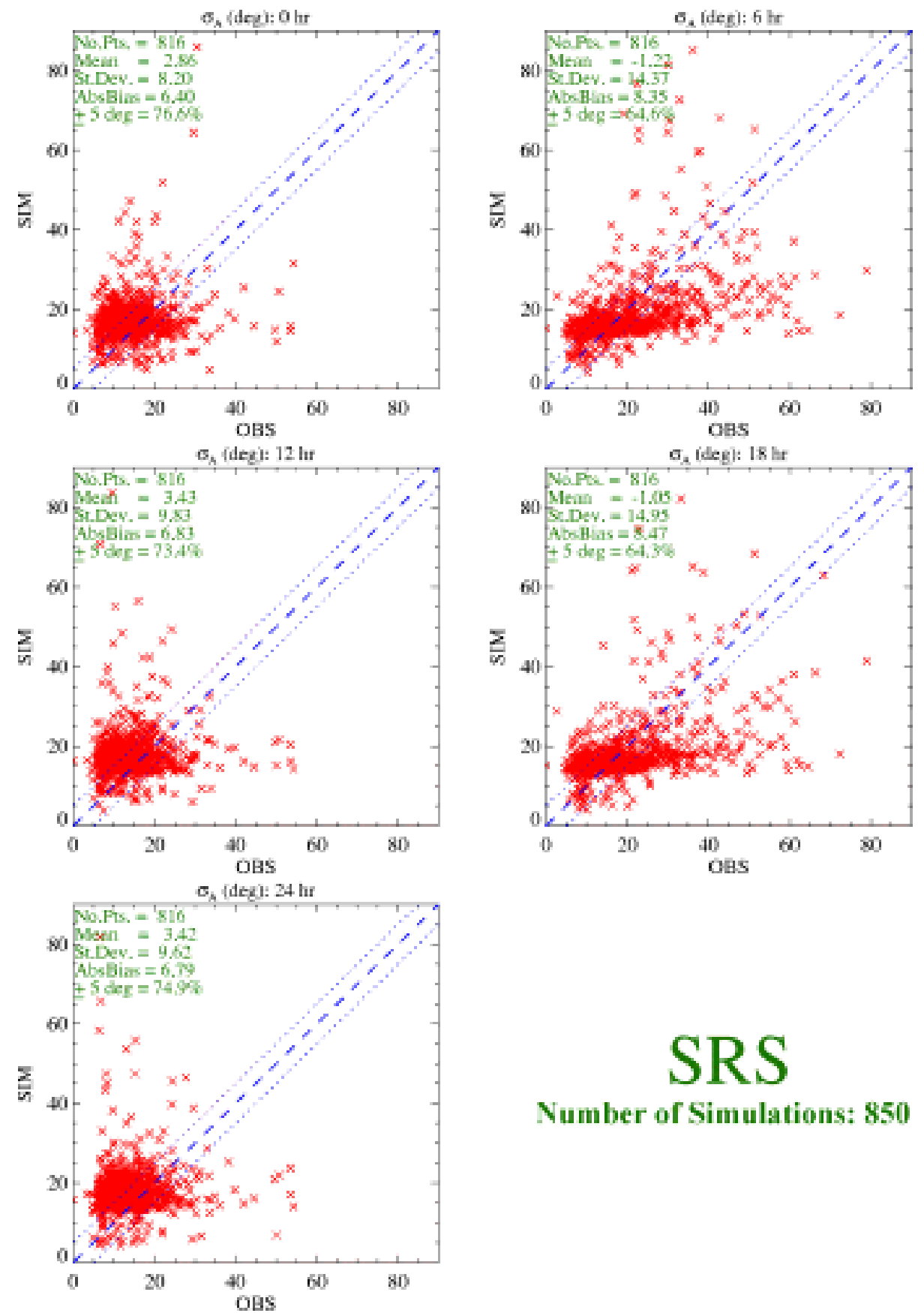

\section{SRS \\ Number of Simulations: 850}

Figure 4: Scatter plot showing surface deviation in azimuth angle (deg) comparisons for the SRS for a forecast period of: (a) 0-hr, (b) 6-hr, (c) 12-hr, (d) 18-hr, (e) 24-hr. The observed quantity is shown along the $x$-axis, and the simulated quantity is shown along the $y$-axis. The bracketing value is $5^{\circ}$ to either side of the $45^{\circ}$ line. For each forecast period, the following are given: "No.Pts.", the number of times represented in the plot, "Mean", the mean difference between simulated and observed $\sigma_{A}$, "St.Dev.", the standard deviation, "AbsBias", the mean absolute bias, and the percentage of times in which the simulated $\sigma_{A}$ was within $\pm 5^{\circ}$ of the observed value. 


\section{Simulation Period: February 1999 - March 2000}
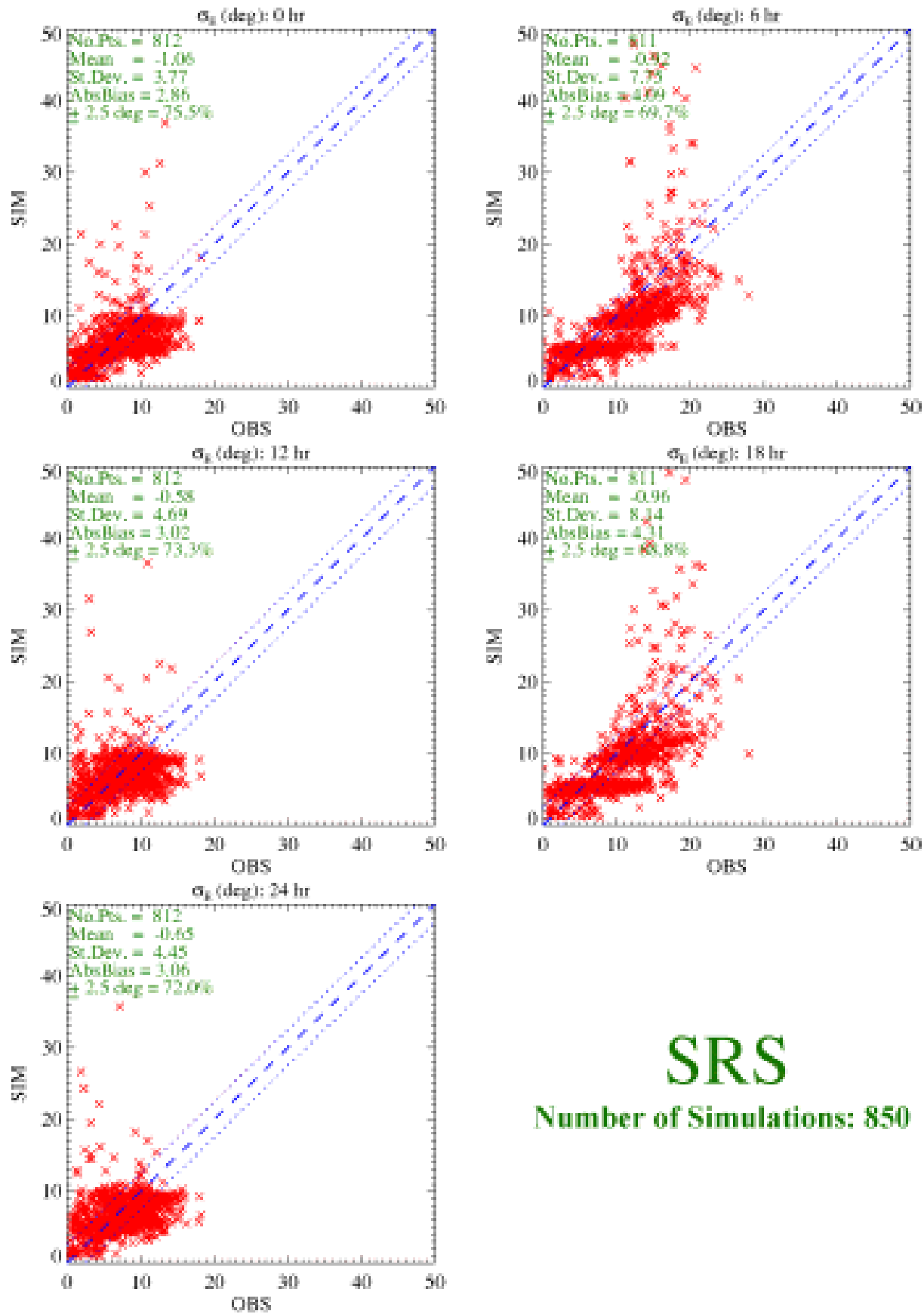

Number of Simulations: $\mathbf{8 5 0}$

Figure 5: Scatter plot showing surface deviation in elevation angle (deg) comparisons for the SRS for a forecast period of: (a) 0-hr, (b) 6-hr, (c) 12-hr, (d) 18-hr, (e) 24-hr. The observed quantity is shown along the $x$-axis, and the simulated quantity is shown along the $y$-axis. The bracketing value is $2.5^{\circ}$ to either side of the $45^{\circ}$ line. For each forecast period, the following are given: "No.Pts.", the number of times represented in the plot, "Mean", the mean difference between simulated and observed $\sigma_{E}$, "St.Dev.", the standard deviation, "AbsBias", the mean absolute bias, and the percentage of times in which the simulated $\sigma_{E}$ was within $\pm 2.5^{\circ}$ of the observed value. 


\section{Simulation Period: April 1998 - March 2000}
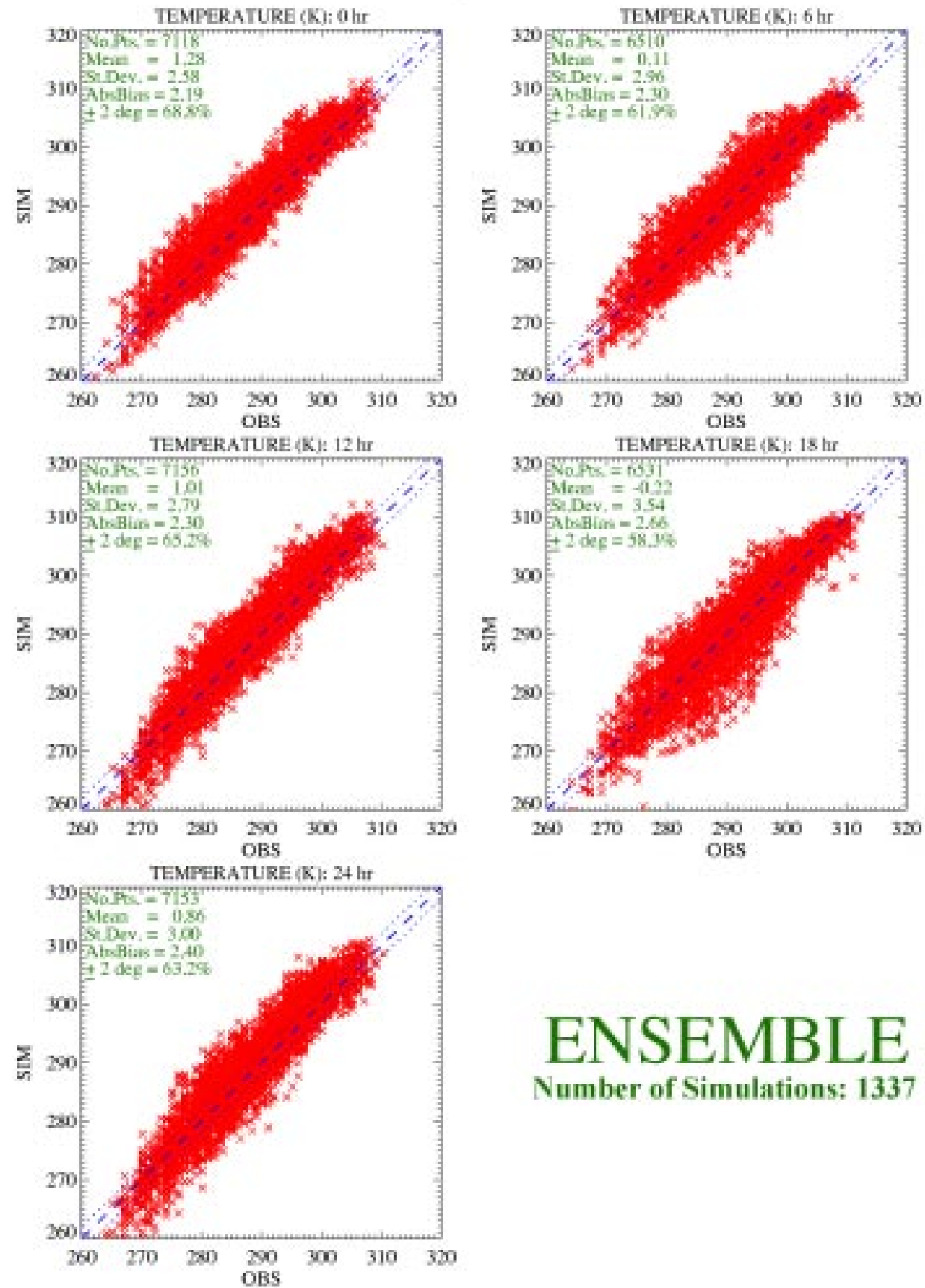

Number of Simulations: 1337

Figure 6: Scatter plot showing surface temperature (K) comparisons for the six locations for a forecast period of: (a) 0-hr, (b) 6-hr, (c) 12-hr, (d) 18-hr, (e) 24-hr. The observed quantity is shown along the $x$-axis, and the simulated quantity is shown along the $y$-axis. The bracketing value is $2 \mathrm{~K}$ to either side of the $45^{\circ}$ line. For each forecast period, the following are shown: "No.Pts.", the number of times represented in the plot, "Mean", the mean difference between simulated and observed temperature, "St.Dev.", the standard deviation, "AbsBias", the mean absolute bias, and the percentage of times in which the simulated temperature was within $\pm 2 \mathrm{~K}$ of the observed value. 
WSRC-TR-2001-00563

December 2001

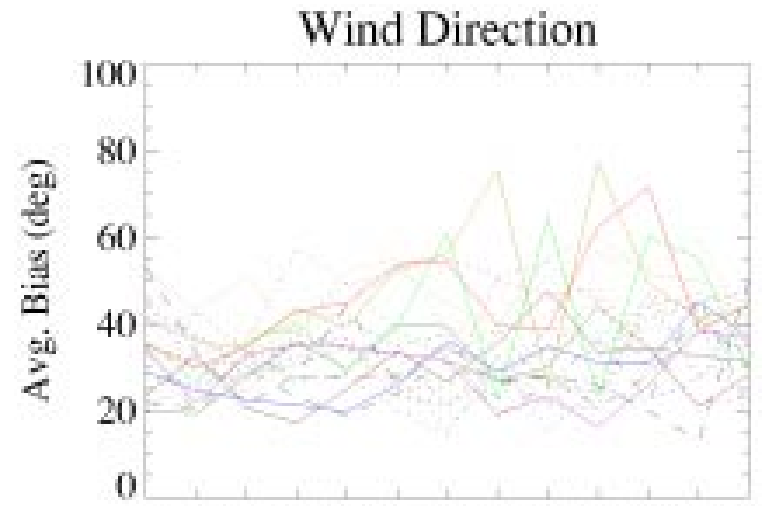

12141618202200020406081012

Time (GMT)

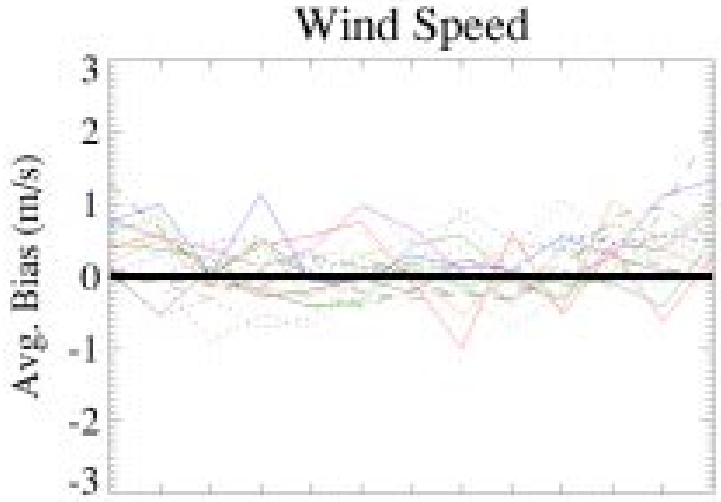

12141618202200020406081012

Time (GMT)

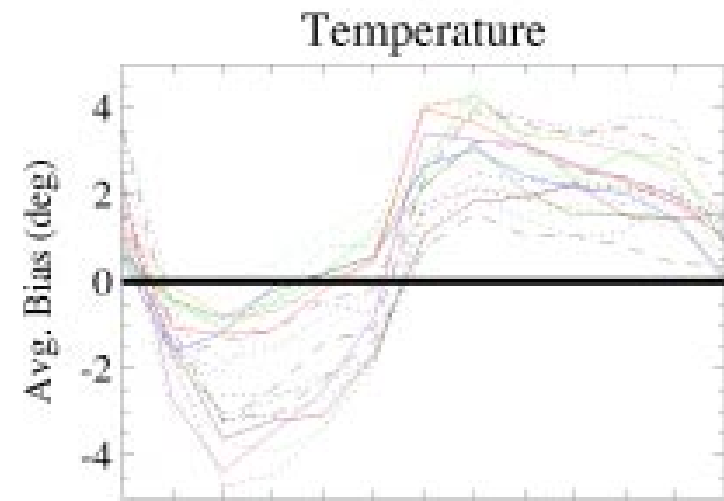

12141618202200020406081012

Time (GMT)

Figure 7: Plots of absolute bias (wind direction) or mean difference (wind speed, temperature) as a function of the time of day in the forecast for Augusta, Georgia. In this case, simulations using the analysis valid for a time of 00 GMT are used to generate a forecast. Note that 12 GMT is an early morning period for this location. Each line represents an average over all simulations within a given month ( 24 months in all) for the resulting difference between simulation and observation. Solid lines are from the year 1998, dotted lines from 1999, and dashed lines from 2000. Note that for the mean differences, a positive number denotes the simulation over-predicted the result. 
WSRC-TR-2001-00563

December 2001

Wind Direction Comparisons (degrees) at the Surface
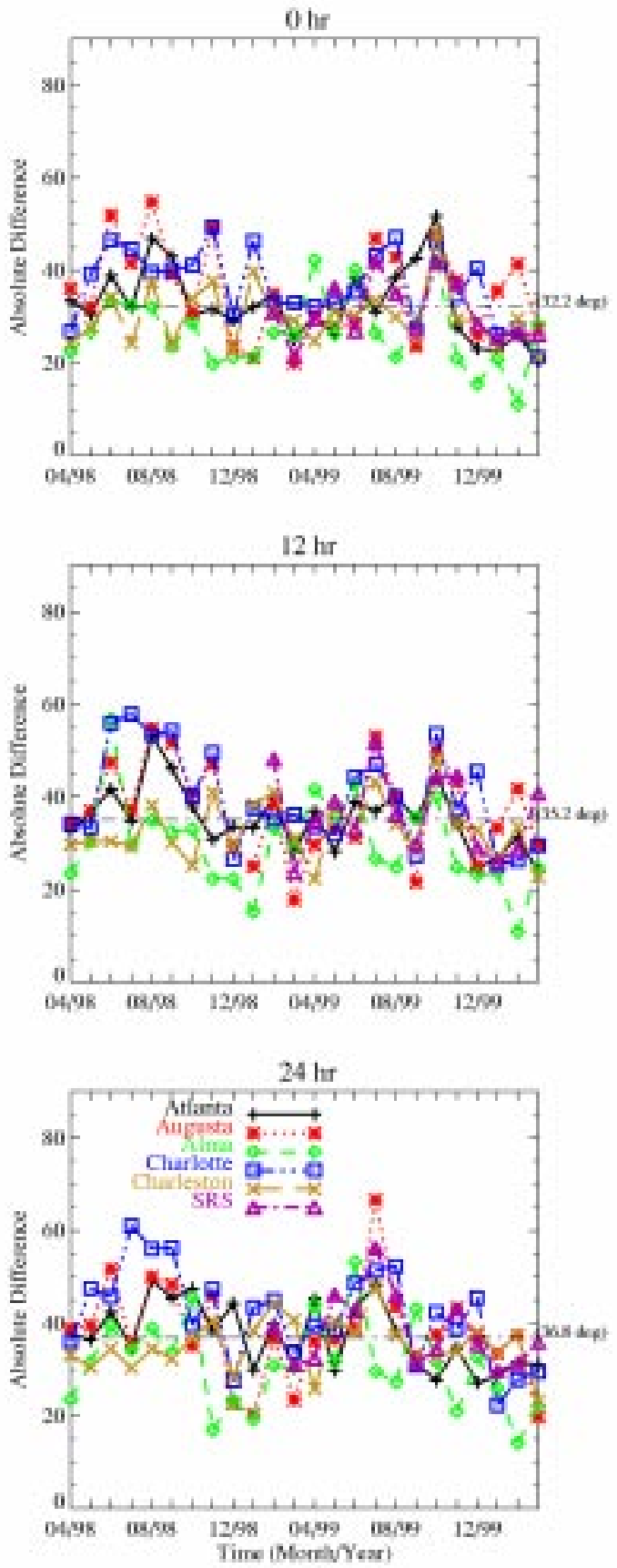

Figure 8: Comparison of simulated and observed wind direction for all 5 cities at the surface for forecast periods of 0, 12, and 24 hours, as well as for the SRS climatology location (from February 1999 onward). Each line in each plot represents a different location, as denoted in the legend. Each symbol represents the average for a particular month, beginning at the far left with April 1998. In this case, the absolute difference in simulation and observation (constrained to $\leq 180^{\circ}$ ) over that month for that particular forecast time is shown. A mean absolute difference is also shown as averaged over all locations and times, with a numerical value given in parentheses near the center of each row. 
WSRC-TR-2001-00563

December 2001

Wind Speed Comparisons $\left(\mathrm{m} \mathrm{s}^{-1}\right)$ at the Surface
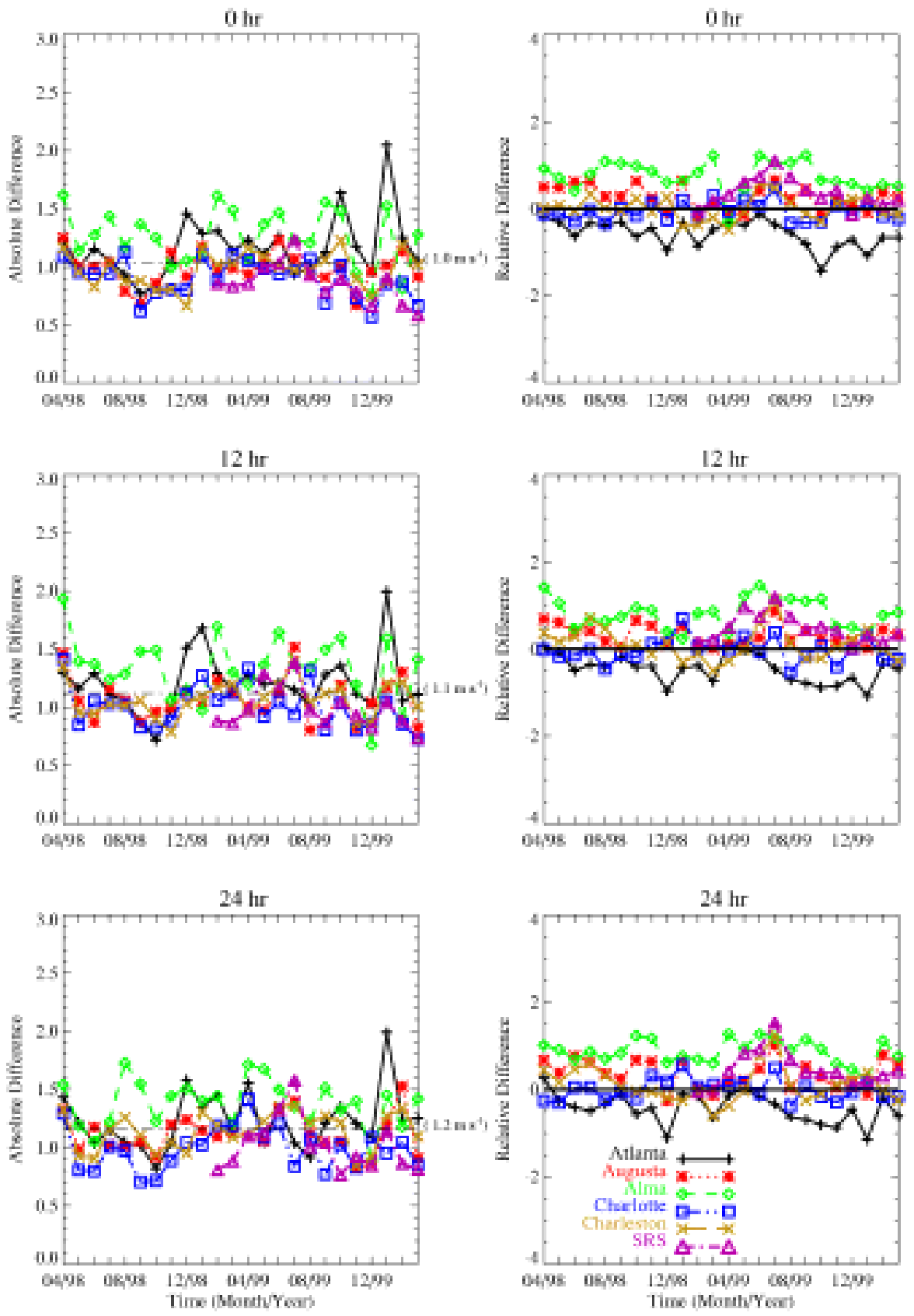

Figure 9: Comparison of simulated and observed wind speed for all 5 cities at the surface for forecast periods of 0 , 12, and 24 hours, as well as for the SRS climatology location (from February 1999 onward). Each line in each plot represents a different location, as denoted in the legend. Each symbol represents the average for a particular month, beginning at the far left with April 1998. The left-hand column shows the absolute difference in simulation and observation over that month for that particular forecast time. The right-hand column shows mean relative differences between simulation and observation. Positive numbers denote the simulation over-predicted the result. A mean absolute difference is also shown for the left-hand column as averaged over all locations and times, with a numerical value given in parentheses near the center of each row. 
Deviation Angle Comparisons (deg) at $26 \mathrm{~m} \mathrm{AGL}$ at SRS
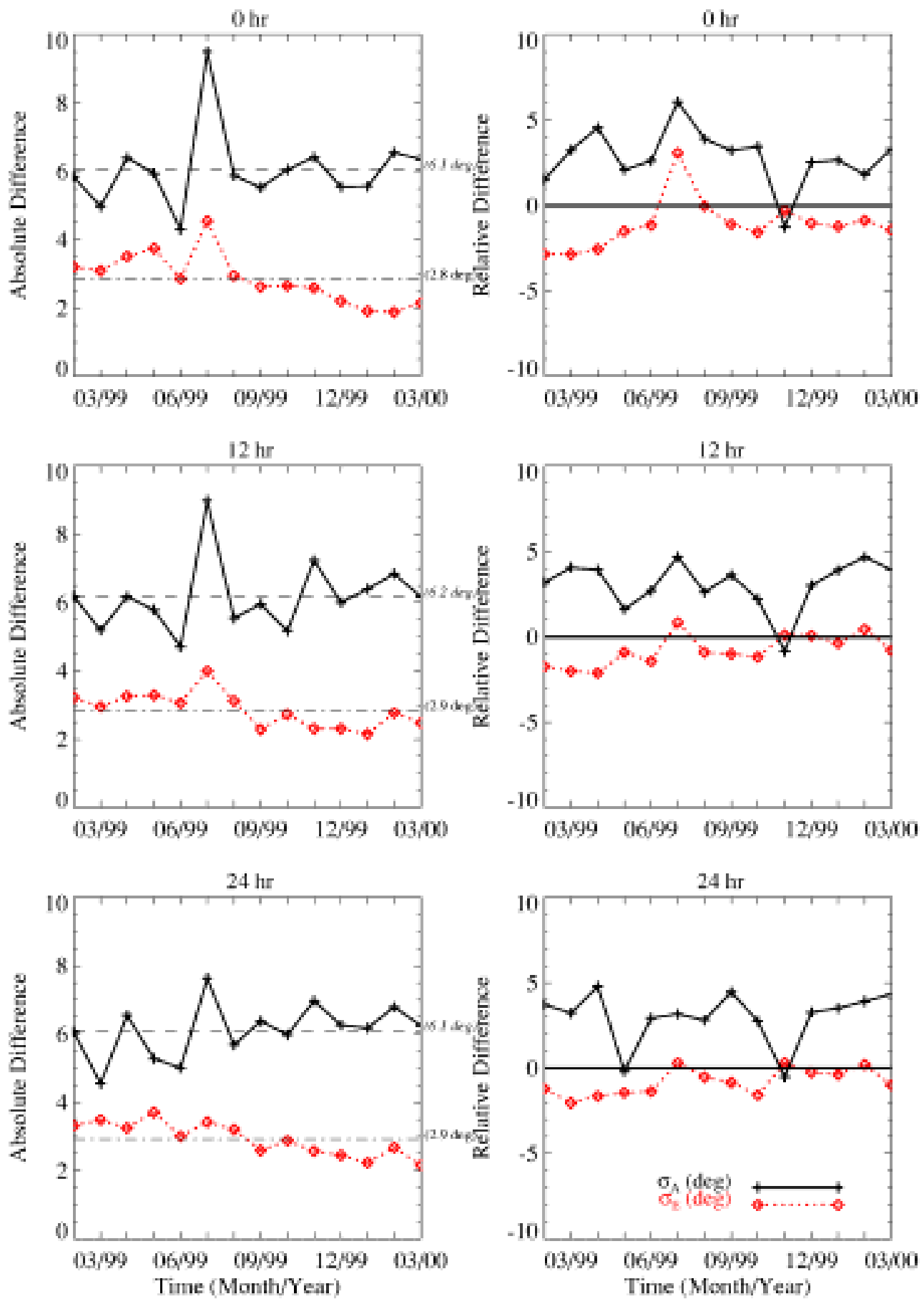

Figure 10: Comparison of simulated and observed deviations in azimuth and elevation angle at the surface at SRS for forecast periods of 0, 12, and 24 hours. Format as in Fig. 9, except the solid line is $\sigma_{A}$ and the dotted line is $\sigma_{E}$.

The mean absolute differences are in italics for $\sigma_{A}$, and in normal text for $\sigma_{E}$. 
WSRC-TR-2001-00563

December 2001

Temperature Comparisons (K) at the Surface
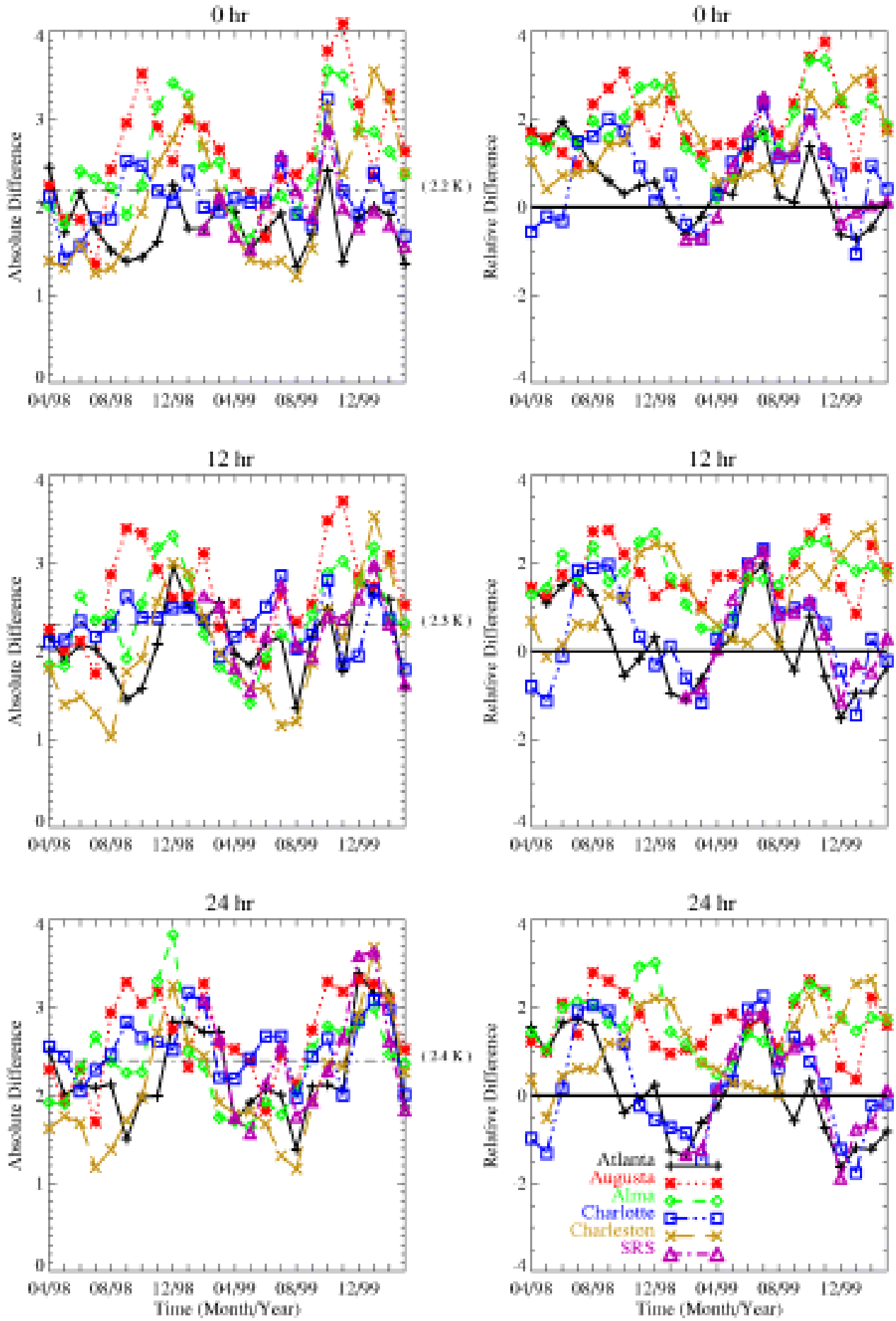

Figure 11: Comparison of simulated and observed temperature for all 5 cities at the surface for forecast periods of 0 , 12, and 24 hours, as well as for the SRS climatology location (from February 1999 onward). Format as in Fig. 9. 
WSRC-TR-2001-00563

December 2001

\section{Wind Direction Comparisons (degrees) at the} $700 \mathrm{mb}$ Pressure Level
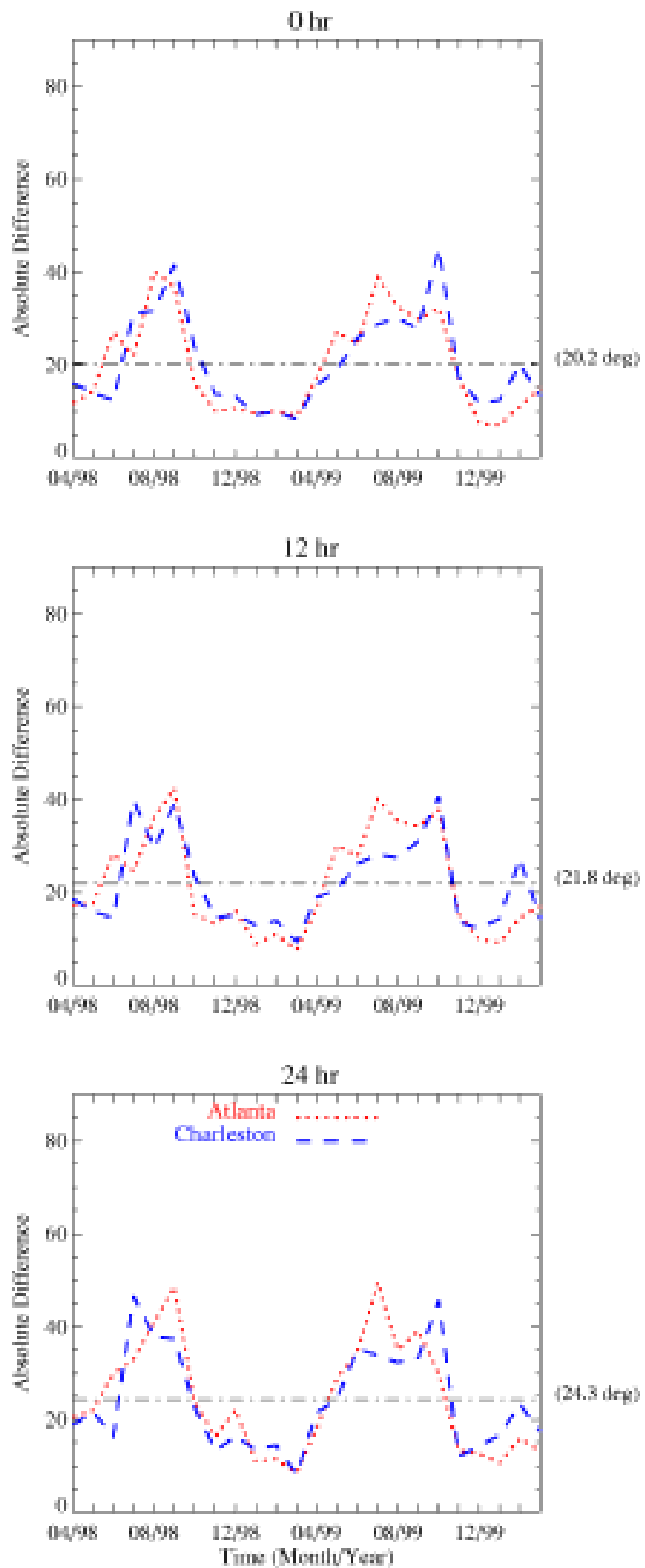

Figure 12: As in Figure 8, except comparisons of simulated and observed wind direction are for Atlanta and Charleston at the 700-mb pressure level, using observations taken from upper-air reports. 
WSRC-TR-2001-00563

December 2001

\section{Wind Speed Comparisons ( $\mathrm{m} \mathrm{s}^{-1}$ ) at the $500 \mathrm{mb}$ Pressure Level}
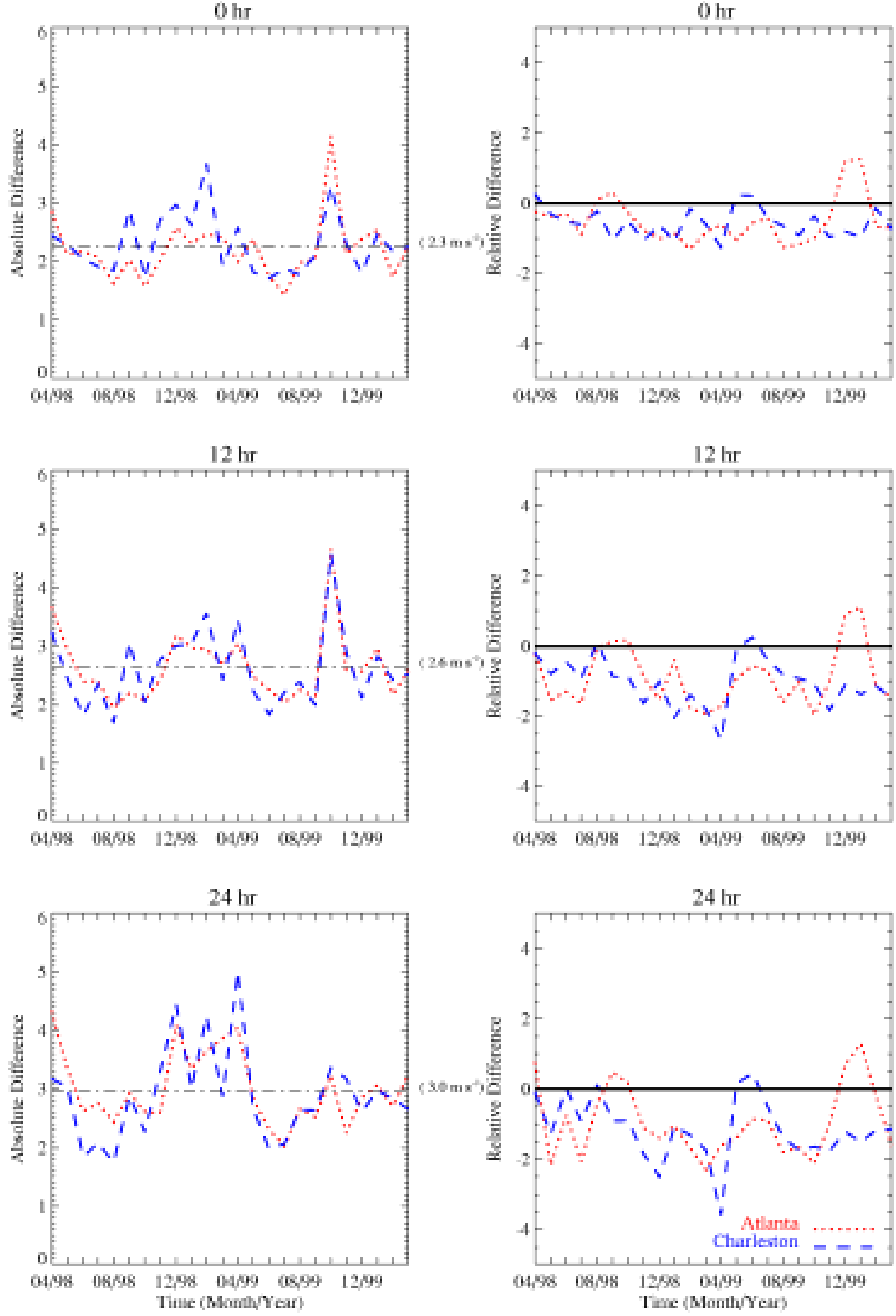

Figure 13: As in Figure 9, except comparisons of simulated and observed wind speed are for Atlanta and Charleston at the 500-mb pressure level, using observations taken from upper-air reports. 
WSRC-TR-2001-00563

December 2001

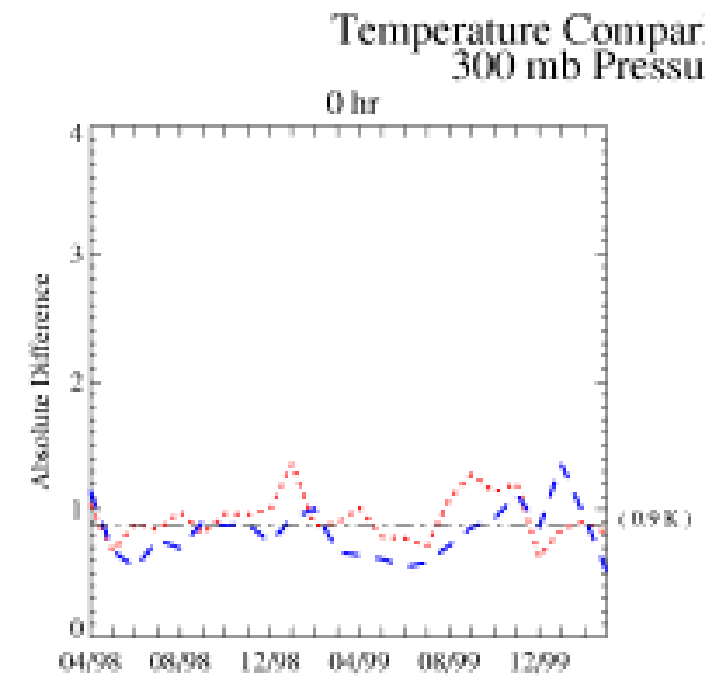

然e
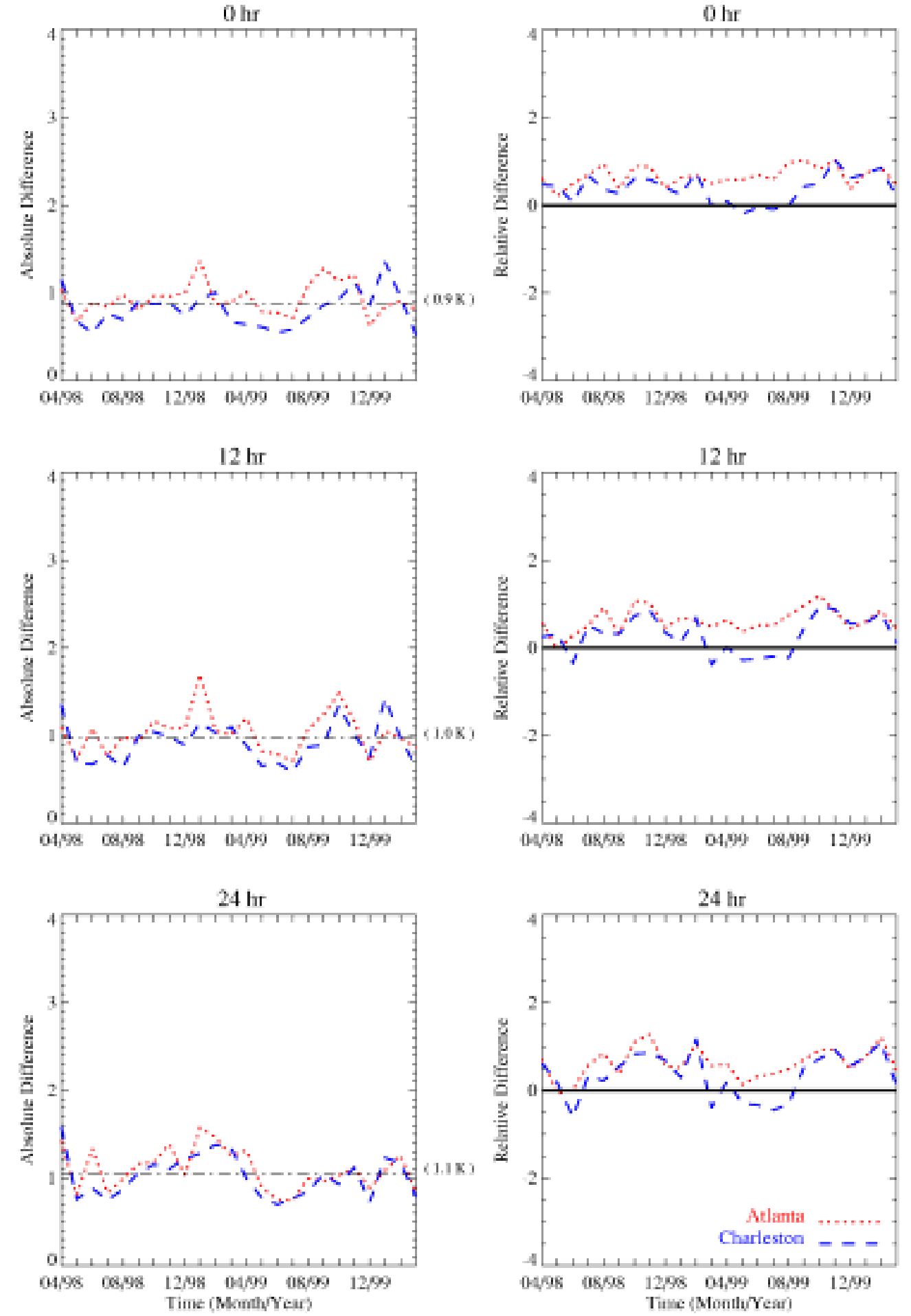

Figure 14: As in Figure 11, except comparisons of simulated and observed temperature are for Atlanta and Charleston at the 300-mb pressure level, using observations taken from upper-air reports. 

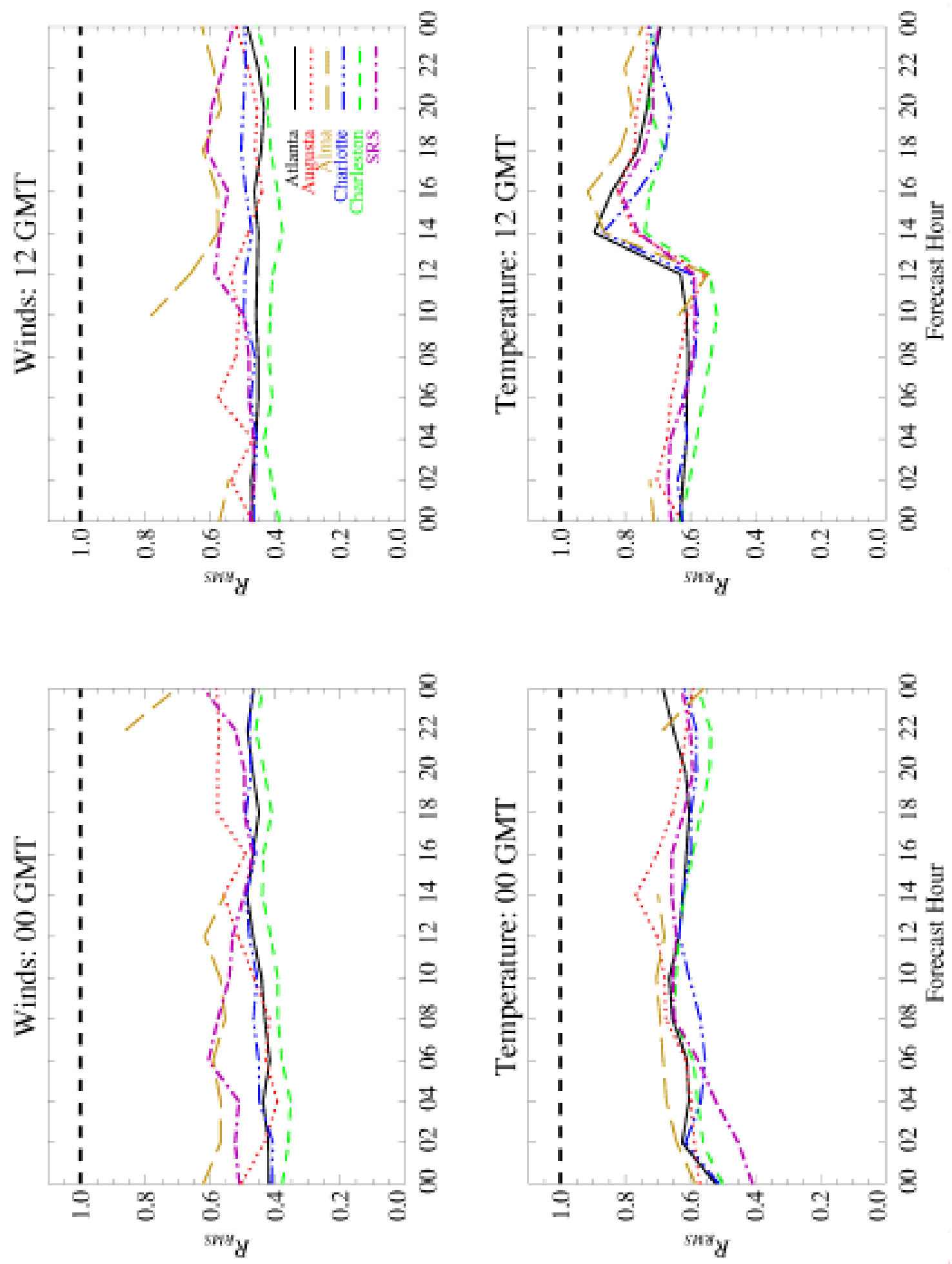

Figure 15: Plots of the ratio of root-mean-square error to observed standard deviation as a function of the forecast time for the six different locations. Each value at a given forecast time is averaged over all months from April 1998 to March 2000. The top panels are for vector winds, while the bottom panels are for temperature. 

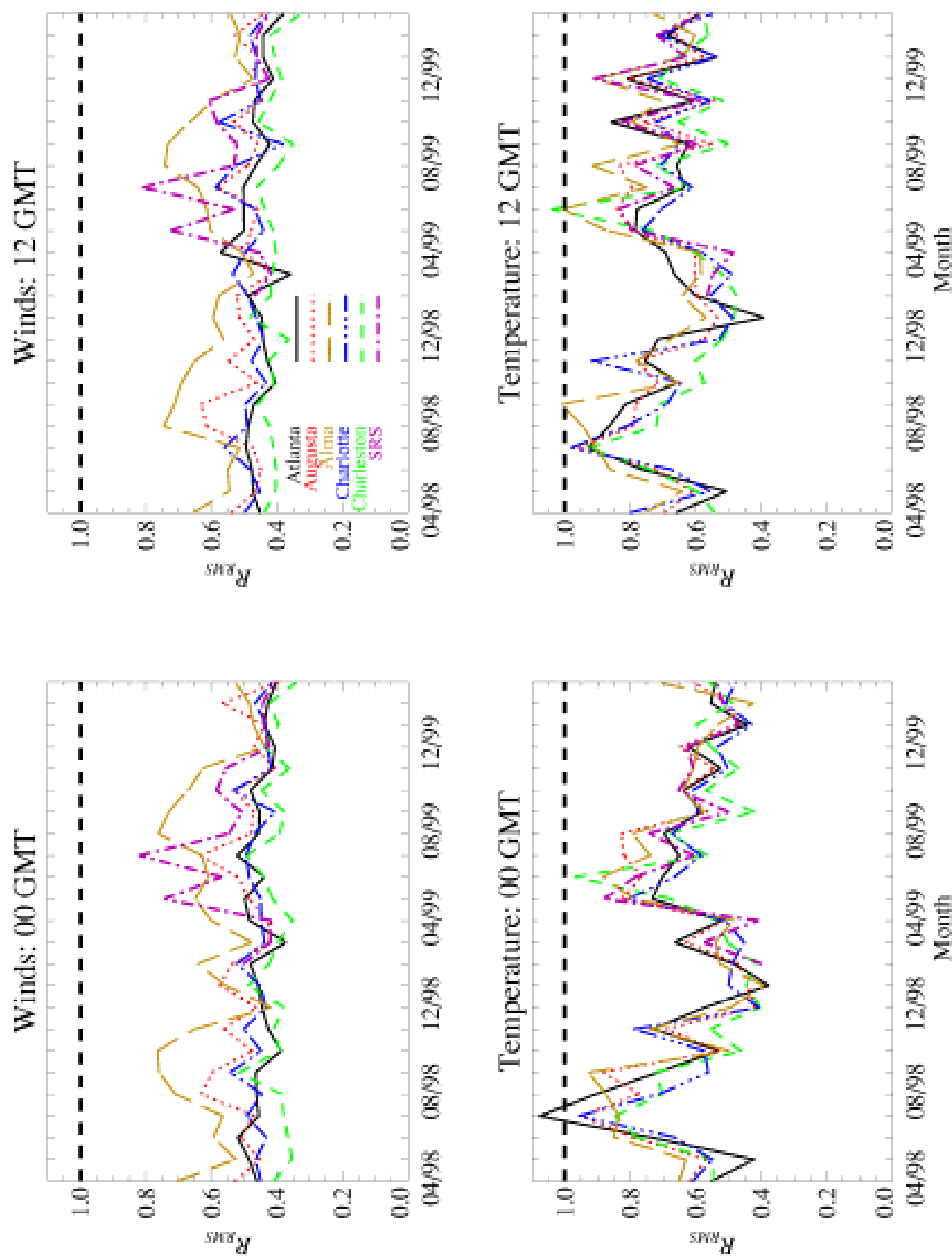

Figure 16: Plots of the ratio of root-mean-square error to observed standard deviation as a function of the month for the six different locations. Each value for a given month is averaged over all forecast times from 0 to 24 hours at 2hr increments. The top panels are for vector winds, while the bottom panels are for temperature. 

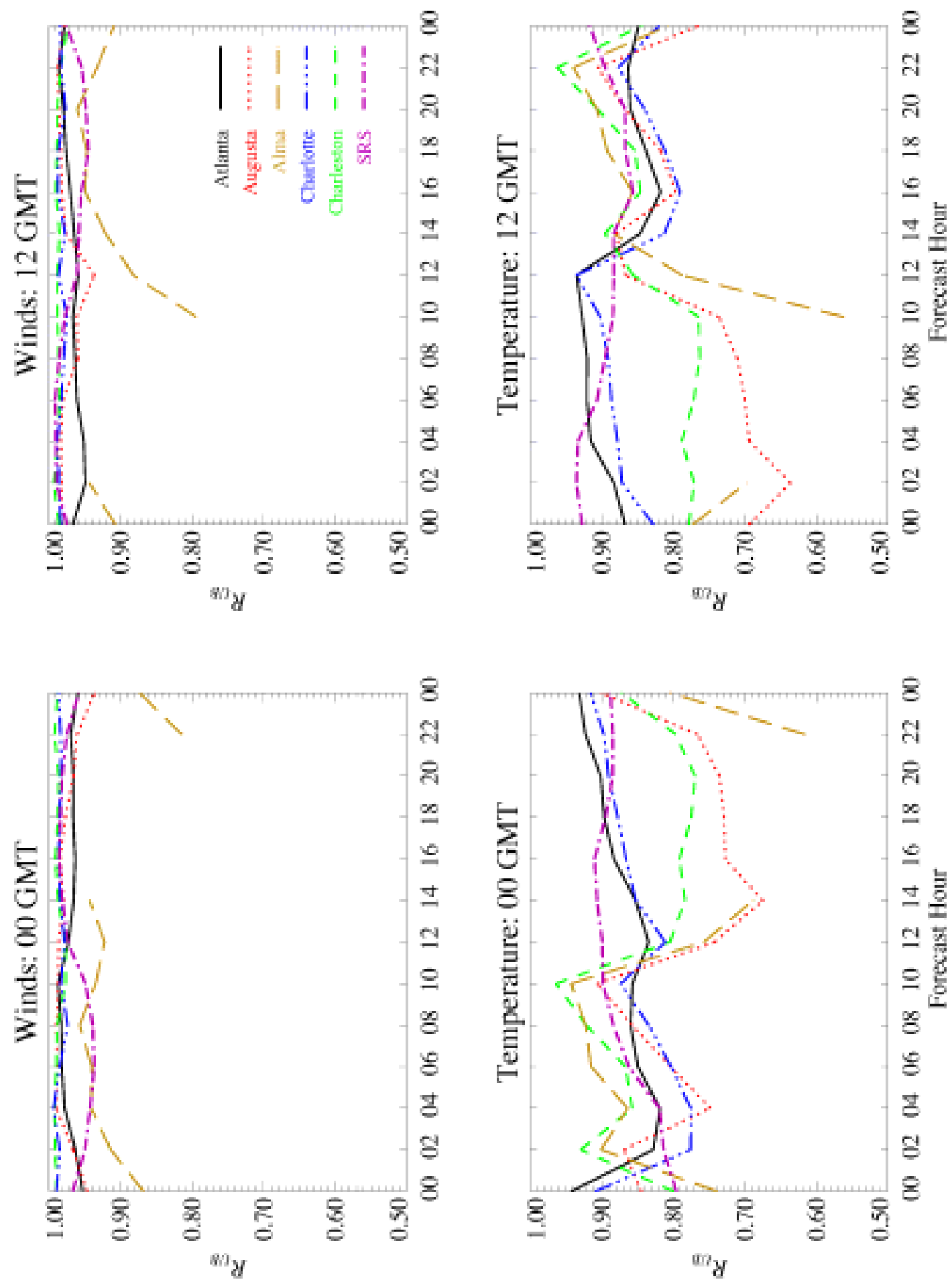

Figure 17: Plots of the ratio of unbiased to biased root-mean-square error as a function of the forecast time for the six different locations. Each value at a given forecast time is averaged over all months from April 1998 to March 2000. The top panels are for vector winds, while the bottom panels are for temperature. 
WSRC-TR-2001-00563

December 2001
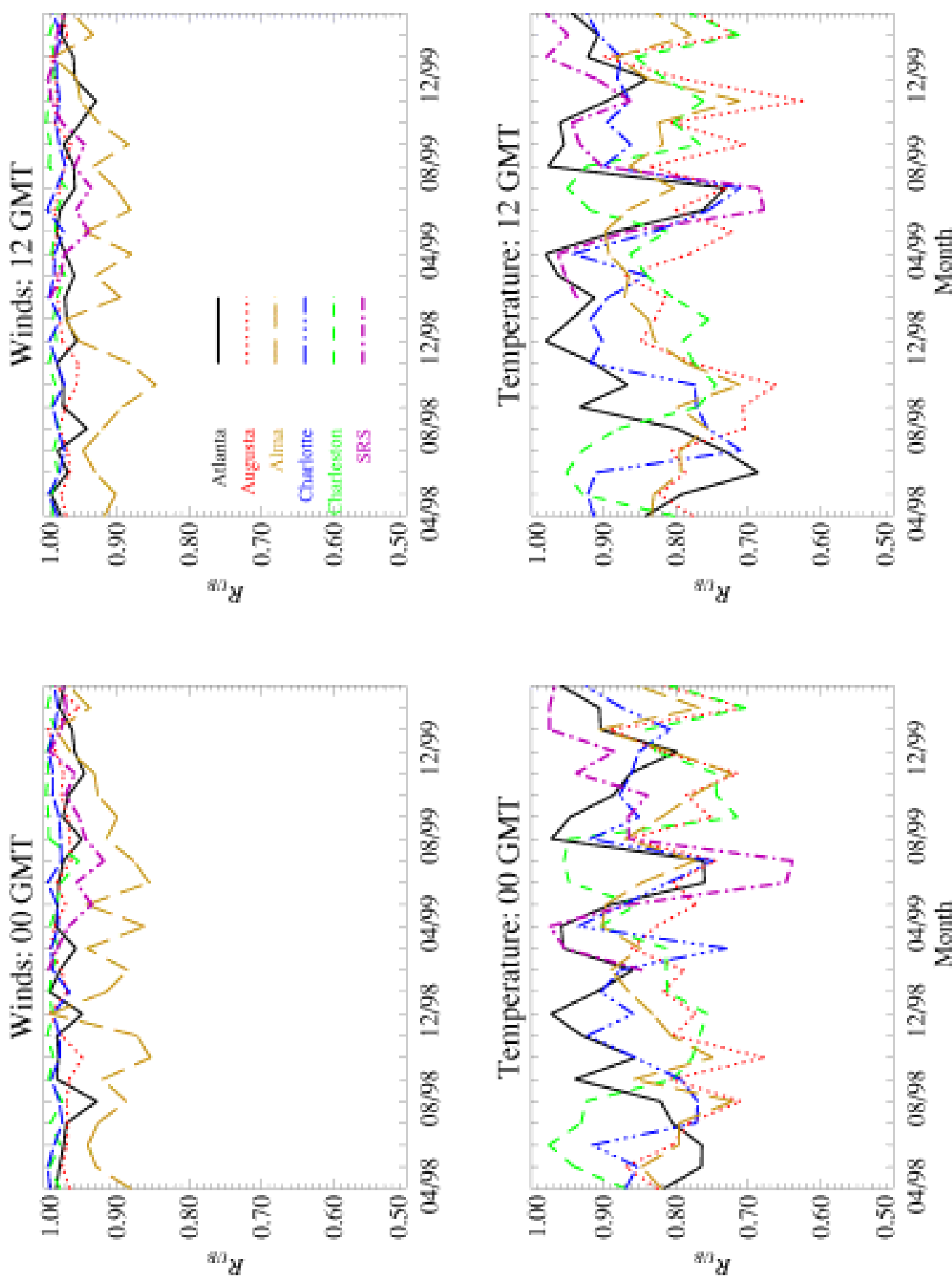

Figure 18: Plots of the ratio of unbiased to biased root-mean-square error as a function of the month for the six different locations. Each value for a given month is averaged over all forecast times from 0 to 24 hours at 2-hr increments. The top panels are for vector winds, while the bottom panels are for temperature. 

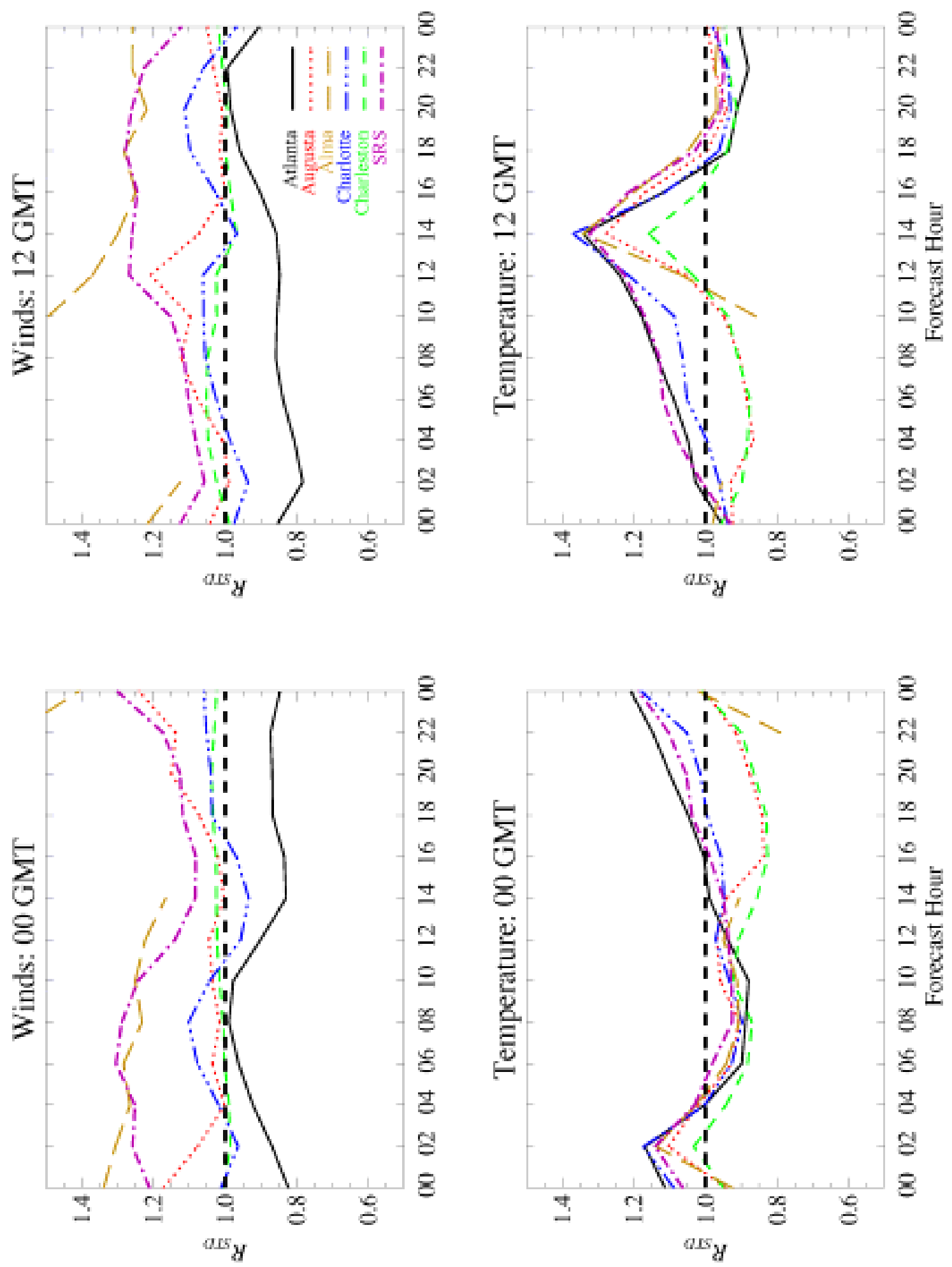

Figure 19: Plots of the ratio of simulated to observed standard deviation as a function of the forecast time for the six different locations. Each value at a given forecast time is averaged over all months from April 1998 to March 2000.

The top panels are for vector winds, while the bottom panels are for temperature. 

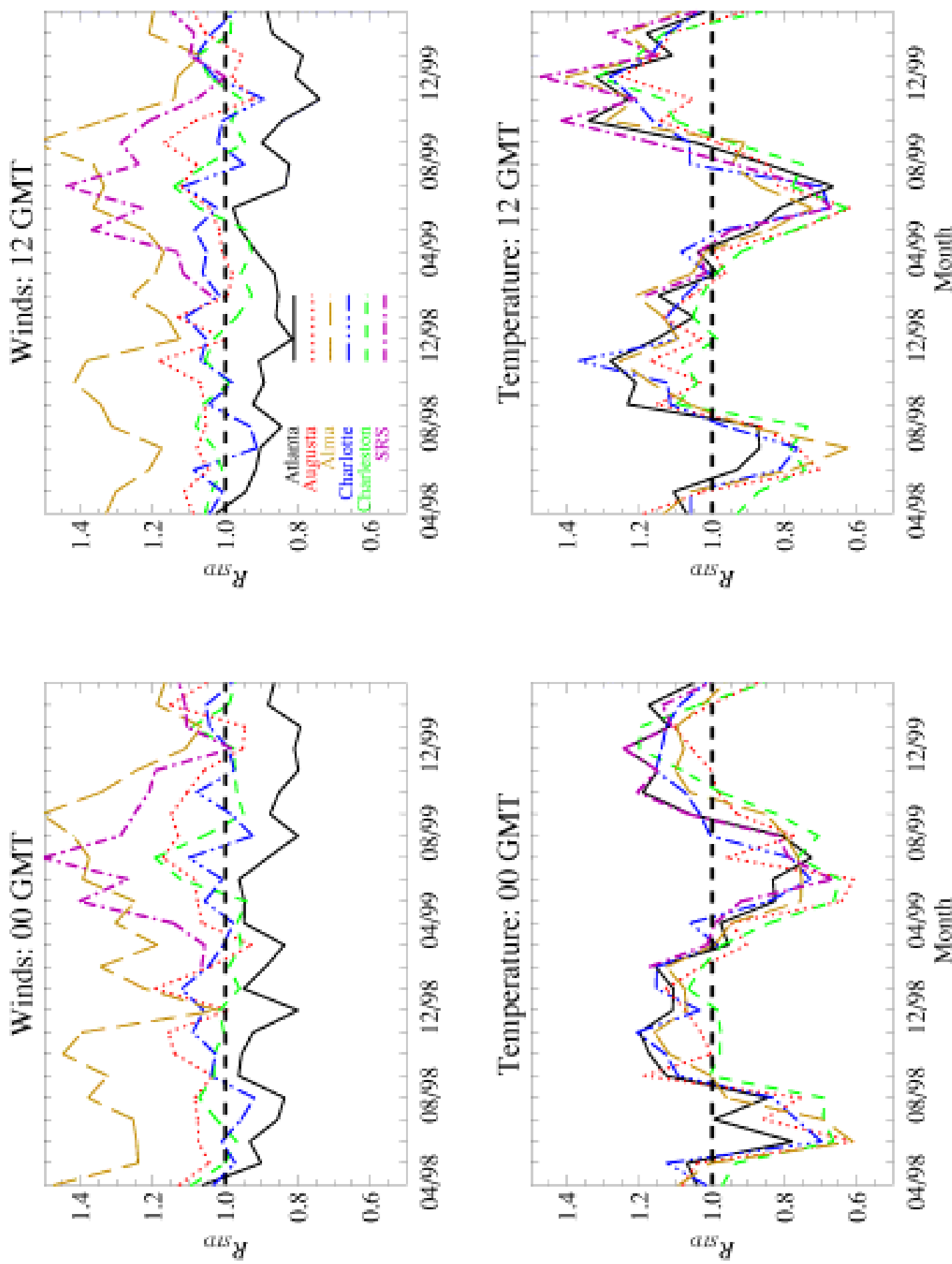

Figure 20: Plots of the ratio of simulated to observed standard deviation as a function of the month for the six different locations. Each value for a given month is averaged over all forecast times from 0 to 24 hours at 2-hr increments. The top panels are for vector winds, while the bottom panels are for temperature. 

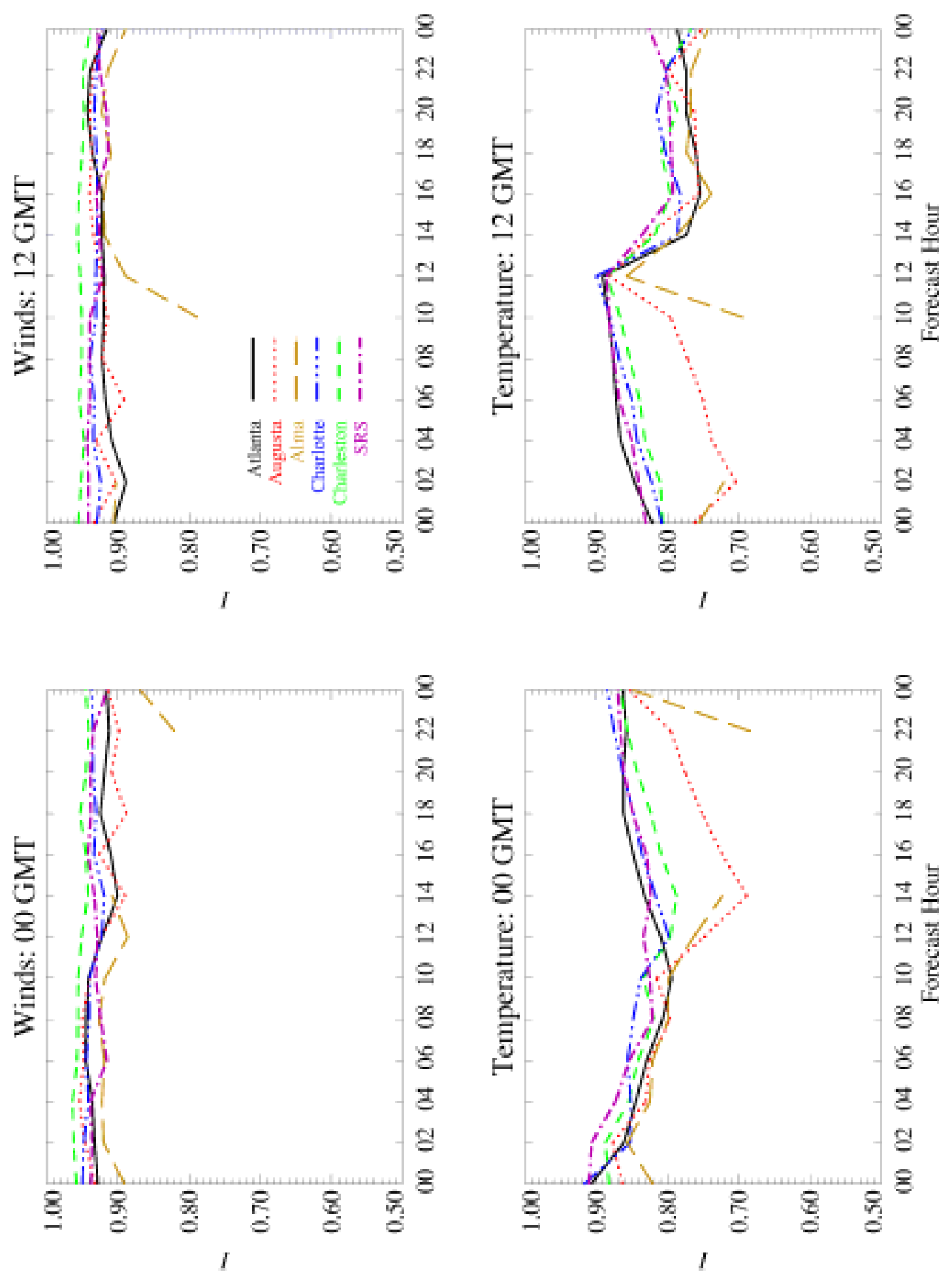

Figure 21: Plots of the index of agreement as a function of the forecast time for the six different locations. Each value at a given forecast time is averaged over all months from April 1998 to March 2000. The top panels are for vector winds, while the bottom panels are for temperature. 
WSRC-TR-2001-00563

December 2001
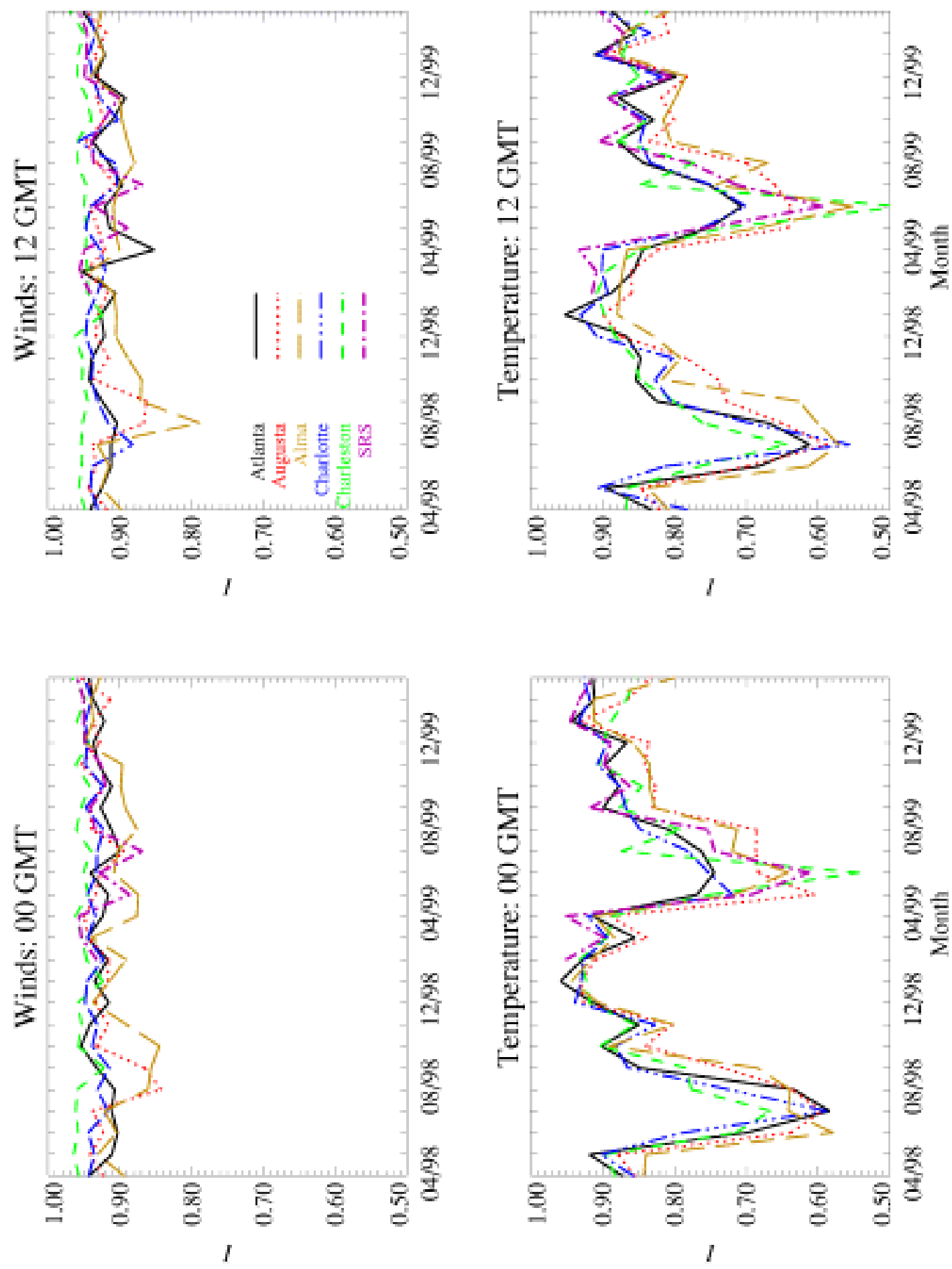

Figure 22: Plots of the index of agreement as a function of the month for the six different locations. Each value for a given month is averaged over all forecast times from 0 to 24 hours at 2 -hr increments. The top panels are for vector winds, while the bottom panels are for temperature. 